Review

\title{
A Systematic Review of Therapeutic Facet Joint Interventions in Chronic Spinal Pain
}

Mark V. Boswell, MD, PhD¹, James D. Colson, MD², Nalini Sehgal, MD³, Elmer E. Dunbar, MD, and Richard Epter, MD

From: ${ }^{1}$ Texas Tech University Health Science Center, Lubbock, TX; ${ }^{2}$ West Virginia University Hospitals, 3University of Wisconsin School of Medicine and Public Health; 4 Pain Control Network PSC, Louisville, KY; ${ }_{5}^{5}$ Augusta Pain Center, Augusta, GA. Dr. Boswell ${ }^{1}$ is Professor of Anesthesiology, Interim Department Chair and Director of the Messer

Racz Pain Center, Department of Anesthesiology, Texas Tech

University Health Sciences Center, Lubbock, TX. Dr. Colson ${ }^{2}$ is Assistant Professor of Anesthesiology, Department of Anesthesiology, Pain

Medicine Service, West Virginia

University Hospitals. Dr. Sehgal ${ }^{3}$ is Medical Director, Interventional Pain

Program, University of Wisconsin School of Medicine and Public Health and Associate Professor Rehabilitation Medicine, Madison,

WI. Dr. Dunbar 4 is Medical Director,

Pain Control Network, Louisville,

KY. Dr. Epter 5 is Medical Director, Augusta Pain Center, Augusta, GA. Address Correspondence: Mark V. Boswell, MD, PhD, Dept. of Anesthesiology, 3601 th St. MS, 8182 , Room 1C 282 Lubbock, TX 79430

E-mail: boswellmv@earthlink.net Funding: None Conflict of interest: None

Free full manuscript: www.painphysicianjournal.com
Background: Facet joints are considered to be a common source of chronic spinal pain. Facet joint interventions, including intraarticular injections, medial branch nerve blocks, and neurotomy (radiofrequency and cryoneurolysis) are used to manage chronic facet-mediated spinal pain. A systematic review of therapeutic facet interventions published in January 2005, concluded that facet interventions were variably effective for short-term and long-term relief of facet joint pain.

Objective: To provide an updated evaluation of the effectiveness of 3 types of facet joint interventions in managing chronic spinal pain.

Study Design: A systematic review utilizing criteria established by the Agency for Healthcare Research and Quality (AHRQ) for evaluation of randomized and non-randomized trials and the Cochrane Musculoskeletal Review Group for randomized trials.

Methods: Data sources included relevant literature of the English language identified through searches of MEDLINE and EMBASE (November 2004 to December 2006) and manual searches of bibliographies of known primary and review articles within the last 2 years. Results of the analyses were performed for the different modes of facet joint interventions for the cervical, thoracic and lumbar spine, to determine short- and long-term outcome measurements and complications associated with these procedures.

Outcome Measures: The primary outcome measure was pain relief. For intraarticular facet joint injections and medial branch blocks, short-term pain relief was defined as relief lasting less than 6 weeks and long-term relief as 6 weeks or longer. For medial branch blocks, repeated injections at defined intervals provided long-term pain relief. For medial branch radiofrequency neurotomy, short-term pain relief was defined as relief lasting less than 3 months and long-term relief as lasting 3 months or longer. Other outcome measures included functional improvement, improvement of psychological status, and return to work.

Results: For cervical intraarticular facet joint injections, the evidence is limited for short- and long-term pain relief. For lumbar intraarticular facet joint injections, the evidence is moderate for short- and long-term pain relief. For cervical, thoracic, and lumbar medial branch nerve blocks with local anesthetics (with or without steroids), the evidence is moderate for short- and longterm pain relief with repeat interventions. The evidence for pain relief with radiofrequency neurotomy of cervical and lumbar medial branch nerves is moderate for short- and long-term pain relief, and indeterminate for thoracic facet neurotomy.

Conclusion: With intraarticular facet joint injections, the evidence for short- and long-term pain relief is limited for cervical pain and moderate for lumbar pain. For medial branch blocks, the evidence is moderate for short- and long-term pain relief. For medial branch neurotomy, the evidence is moderate for short- and long-term pain relief.

Key words: Spinal pain, neck pain, low back pain, facet or zygapophysial joints, intraarticular facet joint injections, medial branch blocks, therapeutic medial branch blocks, radiofrequency neurotomy, cryodenervation.

Pain Physician 2007; 10:229-253 
F acet or zygapophysial joints are considered to be common sources of chronic spinal pain $(1,2)$. In addition to causing localized spinal pain, facet joints may refer pain to adjacent structures. Cervical facet joint pain may radiate to the head, neck, and shoulders. Thoracic facets may produce paraspinous mid-back pain with neuralgic characteristics; and lumbar facet joints may refer pain to the back, buttocks, and proximal lower extremities. Referred pain may assume a pseudoradicular pattern, making the underlying diagnosis difficult to confirm, without the use of diagnostic blocks $(1,2)$.

Facet joints are a well-recognized source of pain in subjects with persistent spinal pain (2). Spinal facet joints have been shown to be a source of pain in the neck and referred pain in the head and upper extremities (3-7); upper back, mid-back, and referred pain in the chest wall $(8,9)$; and the low back and referred pain in the lower extremity (10-15). Facet joints are well innervated by the medial branches of the dorsal rami (16-35). Neuroanatomic, neurophysiologic, and biomechanical studies have demonstrated free and encapsulated nerve endings in facet joints, as well as nerves containing substance $P$ calcitonin gene-related peptide (35-50); facet joint capsules contain low-threshold mechanoreceptors, mechanically sensitive nociceptors and silent nociceptors, (35-53); and lumbar and cervical facet joint capsules can undergo high strains during spine loading $(35,54-65)$.

Consistent with criteria established by the International Association for the Study of Pain (66), facet joints may be a source of chronic pain in $15 \%$ to $45 \%$ of patients with chronic low back pain (67-80); $36 \%$ to $60 \%$ of the patients with chronic neck pain $(68,69,77$ $79,81-86)$; and $34 \%$ to $48 \%$ of the patients with thoracic pain $(78,79,87)$.

Facet joint pain may be managed by intraarticular injections, medial branch blocks, and neurolysis of medial branch nerves. However, conflicting results have been reported for the value of the different treatment modalities in systematic and narrative reviews (88-94). A recent narrative review by Bogduk (94) suggested that intraarticular facet joint injections were no better than placebo for chronic lumbar spine pain. Slipman et al (92), in a review of the evidence for the use of zygapophysial injections and radiofrequency denervation in the treatment of low back pain, found limited evidence for intraarticular injections in the lumbar spine and moderate evidence for radiofrequency neurotomy in the lumbar spine. Boswell et al
(1), in a systematic review of therapeutic facet joint interventions for 3 regions of the spine, showed moderate evidence for lumbar intraarticular facet joint injections for short-term improvement, but only limited evidence for long-term improvement. The evidence was negative for cervical intraarticular facet joint injections. The evidence was moderate for cervical and lumbar medial branch blocks with local anesthetics and steroids. The evidence for pain relief with radiofrequency neurotomy of medial branch nerves was moderate to strong for cervical and lumbar regions.

European guidelines for the management of chronic nonspecific low back pain (90), utilizing the evidence available from January 1995 to November 2002, concluded that intraarticular facet joint injections were ineffective in managing chronic low back pain. In this limited literature review, they showed no significant effectiveness of medial branch blocks. The European guidelines concluded that there was conflicting evidence that radiofrequency denervation of the facet joints is more successful than placebo for eliciting short-term or long-term improvements in pain or functional disability in mechanical chronic low back pain. They also indicated that there was limited evidence that intraarticular denervation of the facet joints is more effective than extraarticular denervation. Geurts et al (88) concluded that there was moderate evidence that radiofrequency lumbar facet denervation was more effective for chronic low back pain than placebo, and there was only limited evidence for effectiveness of radiofrequency neurotomy for chronic cervical zygapophysial joint pain after flexion/extension injury. However, Geurts et al (88) included both medial branch neurotomy and intraarticular neurotomy in their evaluation, along with dorsal root denervation. Manchikanti et al (91) evaluated medial branch neurotomy for the management of chronic spinal pain utilizing the Agency for Healthcare Research and Quality (AHRQ) criteria with inclusion of randomized and observational reports, and concluded that there was strong evidence for short-term relief and moderate evidence for longterm relief of facet joint pain. Niemisto et al (89), in a systematic review of radiofrequency denervation for neck and back pain within the framework of Cochrane Collaboration Back Review Group, concluded that there was limited evidence that radiofrequency denervation had a positive short-term effect on chronic cervical zygapophysial joint pain, and a conflicting short-term effect on chronic low back pain. 
Although there have not been reviews on the therapeutic effectiveness of facet interventions in the past 2 years, there have been several randomized and observational studies of therapeutic interventions.

This systematic review was undertaken to update the effectiveness of intraarticular facet joint blocks, medial branch blocks, and medial branch radiofrequency denervation in the treatment of chronic spinal pain of facet joint origin.

\section{Methods}

\section{Literature Search}

The literature search included MEDLINE and EMBASE (November 2004 - December 2006), Cochrane database, systematic reviews, narrative reviews, cross-references to the reviews and various published trials, and peer-reviewed abstracts from scientific meetings during the past 2 years, published in the English language. The same search strategy was also performed using the Google search engine. The search strategy consisted of facet (zygapophysial joint) injections with local anesthetics and steroids, medial branch facet nerve blocks with local anesthetics and steroids, medial branch (facet nerve) nerve blocks, and radiofrequency neurotomy or cryoneurolysis. The emphasis of the analysis was on chronic spinal pain of facet joint origin.

\section{Selection Criteria and Data Extraction}

This review evaluated randomized and observational studies and reports of complications. To be included in this review, studies should have documented the existence of spinal pain of facet origin using controlled diagnostic facet joint or nerve blocks. However, due to the scarcity of such studies, studies with single blocks were also considered.

Three types of facet interventions were included in this review: intraarticular facet joint injections, medial branch blocks, and medial branch radiofrequency neurotomy, cryoneurolysis, and other neurolytic techniques. All studies providing appropriate management with outcome evaluations of at least 3 months and statistical analysis were reviewed. The primary outcome measure was pain relief at various time points. The secondary outcome measures were functional or psychological improvement, return to work, and complications. Principles of evidence synthesis in interventional pain management for interventional techniques has been described $(95,96)$. For this systematic review, the same principles utilized in the previous systematic review (1) were used.

For evaluating the quality of individual articles, we used the criteria from AHRQ (97). For inclusion, studies must have met at least $50 \%$ of the key domains and elements. Criteria described by the Cochrane Review Group for Musculoskeletal Disorders (98) were also used for evaluation of randomized trials.

Each study was evaluated for inclusion criteria, the study population, outcomes data, and statistical analysis (99). Parameters and criteria are described elsewhere $(1,96)$.

\section{Analysis of Evidence}

Qualitative analysis was conducted using five levels of evidence for effectiveness as illustrated in Table 1. For intraarticular injections and medial branch blocks, pain relief was evaluated for short-term $(<6$ weeks) and long-term (6 weeks or longer) benefit. For neurolytic interventions, short-term relief pain relief was considered to be $<3$ months duration and $\geq 3$ months for long-term benefit.

For randomized trials, a study was judged to be positive if the facet joint intervention was more effective than the reference treatment. For observational studies, results were considered positive if the treatment was effective by defined criteria (e.g., $>50 \%$ pain relief) for the designated period of time. All other conclusions were considered negative. If, in the opinion of the reviewers there were inconsistencies in the conclusions, disagreements were resolved by discussion and consensus.

\section{Results}

\section{Intraarticular Facet Joint Blocks}

Our search strategy ([facet or zygapophysial] AND injection) identified 44 new references; 2 studies met inclusion criteria for the present review $(100,103)$. The search performed for our previous systematic review identified a total of 235 articles, with 10 relevant articles for further review (104-113).

\section{Methodological Quality}

Five randomized studies of intraarticular lumbar steroid facet joint injections $(100,104-107)$ and 1 randomized study of the cervical spine (108) compared results to those of similar groups not receiving intraarticular steroids. Of these, Fuchs et al (100), Carrette et al (104), and Barnsley et al (108) met inclusion criteria (Table 2).

Other randomized trials by Lilius et al (105), Marks 
Table 1. Designation of levels of evidence.

\begin{tabular}{||l|l||}
\hline Level I & $\begin{array}{l}\text { Conclusive: Research-based evidence with multiple relevant and high-quality scientific studies or consistent reviews } \\
\text { of meta-analyses. }\end{array}$ \\
\hline Level II & $\begin{array}{l}\text { Strong: Research-based evidence from at least 1 properly designed randomized, controlled trial; or research-based } \\
\text { evidence from multiple properly designed studies of smaller size; or multiple low quality trials. }\end{array}$ \\
\hline Level III & $\begin{array}{l}\text { Moderate: } \\
\text { a) Evidence obtained from well-designed pseudorandomized controlled trials (alternate allocation or some other } \\
\text { method); } \\
\text { b) Evidence obtained from comparative studies with concurrent controls and allocation not randomized (cohort } \\
\text { studies, case-controlled studies, or interrupted time series with a control group); } \\
\text { c) Evidence obtained from comparative studies with historical control, 2 or more single-arm studies, or interrupted } \\
\text { time series without a parallel control group. }\end{array}$ \\
\hline Level IV & $\begin{array}{l}\text { Limited: Evidence from well-designed nonexperimental studies from more than 1 center or research group; or } \\
\text { conflicting evidence with inconsistent findings in multiple trials. }\end{array}$ \\
\hline Level V & $\begin{array}{l}\text { Indeterminate: Opinions of respected authorities, based on clinical evidence, descriptive studies, or reports of expert } \\
\text { committees. }\end{array}$ \\
\hline
\end{tabular}

Adapted from refs 1,95,96

et al (106), and Nash (107) did not meet inclusion criteria and were excluded. Marks et al (106) and Nash (107) compared the effects of intraarticular injections with medial branch blocks. Even though the number of patients included was of clinical significance with 86 and 67, respectively, patient selection failed to include controlled diagnostic blocks, there was not a blinded evaluation by an independent observer, and the authors utilized poor assessment tools. Lilius et al (105) included patients with neurological deficits, failed to confirm the diagnosis, and used excessive volumes ( 3 $\mathrm{mL}$ to $8 \mathrm{~mL}$ ) of active agents.

Among the observational reports, 7 studies (103, 109-115) met inclusion criteria. Six prospective (103, $109-111,114,115)$ and 2 retrospective evaluations were included $(112,113)$. Of these, 4 prospective studies evaluated low back pain (109-111,114), 2 prospective evaluations were performed in the neck $(103,115)$ and 2 retrospective evaluations evaluated chronic low back pain $(112,113)$.

Gorbach et al (101) evaluated 42 consecutive patients meeting diagnostic criteria for lumbar facet syndrome by history or examination, but performed intraarticular facet joint injections on one occasion, under fluoroscopic guidance. This study was excluded from further analysis in the present review due to only a 3-month follow-up.

Shih and colleagues (102) reported the results of lumbar intraarticular facet joint injections in a prospective study of 277 patients with low back pain. Following injections of lidocaine, betamethasone, and contrast, positive responses (VAS < 5) were noted in $72.1 \%(147 / 204)$ of patients after 3 weeks, $40.7 \%$ (83/204) after 6 weeks, and 31.4\% (64/204) after 12 weeks. The injections were done for diagnostic purposes and the authors noted modest pain relief as described above. However, since the study was designed primarily to test the diagnostic value and clinical benefit of intraarticular injections and because follow-up was limited to 3 months, the study was excluded from further analysis.

\section{Study Characteristics}

Three randomized studies, those by Fuchs et al (100) and Carette et al (104) involving lumbar facet joint injections, and by Barnsley et al (108) involving cervical facet joint injections, are considered high quality and have been used in the evidence synthesis. Details of these randomized trials are shown in Table 2. Even though methodological criteria for both the above studies were considered optimal, Carette et al (104) failed to exclude placebo responders, which may account for the relatively high incidence of pa- 
Table 2. Characteristics of published randomized trials of intraarticular facet joint injections.

\begin{tabular}{|c|c|c|c|c|c|}
\hline Study/Methods & Participants & Intervention(s) & Outcome(s) & Result(s) & $\begin{array}{c}\text { Conclusion(s) } \\
\text { Short-term } \\
\text { relief }<6 \mathrm{wks} \\
\text { Long-term } \\
\text { relief } \geq 6 \mathrm{wks}\end{array}$ \\
\hline \multicolumn{6}{|l|}{ Lumbar Spine } \\
\hline $\begin{array}{l}\text { Fuchs et al (100) } \\
\text { Lumbar facet joint } \\
\text { injections } \\
\text { Randomized, } \\
\text { controlled, blind } \\
\text { observer, clinical } \\
\text { study } \\
\text { AHRQ score: } 9 / 10 \\
\text { Cochrane score: } 9 / 10\end{array}$ & $\begin{array}{l}60 \text { patients with } \\
\text { chronic non-radicular } \\
\text { lumbar pain. Patients } \\
\text { were randomly } \\
\text { assigned to } 2 \text { groups } \\
\text { to receive } 10 \mathrm{mg} \text { of } \\
\text { sodium hyaluronate } \\
\text { (SH) or } 10 \mathrm{mg} \\
\text { of triamcinolone } \\
\text { acetonide; TA. }\end{array}$ & $\begin{array}{l}\text { The facet joints on } \\
\text { both sides at levels } \\
\text { L3/4, L4/5, and } \\
\text { L5/S1 were treated } \\
\text { once per week } \\
\text { under computed } \\
\text { tomographic } \\
\text { guidance, with } \\
10 \text { mg of sodium } \\
\text { hyaluronate, or } 10 \\
\text { mg of triamcinolone } \\
\text { acetonide. }\end{array}$ & $\begin{array}{l}\text { Follow-up: } 3 \text { and } \\
6 \text { months outcome } \\
\text { parameters: } \\
\text { visual analog scale, } \\
\text { Roland Morris } \\
\text { questionnaire, Os- } \\
\text { westry Disability } \\
\text { questionnaire, Low } \\
\text { Back Outcome } \\
\text { Score, Short Form } \\
36 \text { questionnaire. }\end{array}$ & $\begin{array}{l}\text { Significant pain relief } \\
\text { and improved function } \\
\text { and quality of life were } \\
\text { observed with both } \\
\text { treatments. The study } \\
\text { results were positive } \\
\text { for both hyaluronate } \\
\text { and glucocorticoid } \\
\text { injections. }\end{array}$ & $\begin{array}{l}\text { Positive short- } \\
\text { term and long- } \\
\text { term relief }\end{array}$ \\
\hline $\begin{array}{l}\text { Carrette et al (104) } \\
\text { Lumbar facet joint } \\
\text { injections } \\
\text { Randomized, } \\
\text { double-blind, } \\
\text { placebo-controlled } \\
\text { trial } \\
\text { AHRQ score: } 10 / 10 \\
\text { Cochrane score: } 10 / 10\end{array}$ & $\begin{array}{l}190 \text { patients entered } \\
\text { Phase I of the } \\
\text { study. Of these, } 110 \\
\text { (58\%) reported a } \\
\text { reduction of } 50 \% \text { or } \\
\text { more in their pain } \\
\text { after the injections } \\
\text { of lidocaine. } 101 \\
\text { patients entered the } \\
\text { randomized trial } \\
\text { (Phase II), } 51 \text { in the } \\
\text { methylprednisolone } \\
\text { group, and } 50 \text { in the } \\
\text { placebo group. }\end{array}$ & $\begin{array}{l}\text { The patients received } \\
\text { injections of either } \\
20 \mathrm{mg}(1 \mathrm{~mL}) \text { of } \\
\text { methylprednisolone } \\
\text { acetate mixed with } \\
1 \mathrm{~mL} \text { of isotonic } \\
\text { saline or } 2 \mathrm{~mL} \text { of } \\
\text { isotonic saline in each } \\
\text { of the facet joints. } \\
\text { All the injections } \\
\text { were preceded by } \\
\text { arthrography and } \\
\text { were performed under } \\
\text { fluoroscopic guidance. }\end{array}$ & $\begin{array}{l}95 \text { patients of } 101 \\
\text { were followed for } \\
6 \text { months and their } \\
\text { condition assessed } \\
\text { with scales of } \\
\text { pain severity, } \\
\text { back mobility, } \\
\text { and limitation of } \\
\text { function. }\end{array}$ & $\begin{array}{l}42 \% \text { of the patients who } \\
\text { received methylprednis- } \\
\text { olone and } 33 \% \text { of those } \\
\text { who received placebo } \\
\text { reported marked or } \\
\text { very marked improve- } \\
\text { ment with no significant } \\
\text { difference among the } \\
\text { groups at } 3 \text { months (ver- } \\
\text { sus } 15 \% \text { control group); } \\
\text { at } 6 \text { months increased } \\
\text { to } 46 \% \text {, with statistically } \\
\text { significant difference (p } \\
=0.002 \text { ). }\end{array}$ & $\begin{array}{l}\text { Negative short- } \\
\text { term and long- } \\
\text { term relief }\end{array}$ \\
\hline \multicolumn{6}{|l|}{ Cervical Spine } \\
\hline $\begin{array}{l}\text { Barnsley et al (108) } \\
\text { Cervical facet joint } \\
\text { injections } \\
\text { Randomized, } \\
\text { double-blind } \\
\text { AHRQ score: } 10 / 10 \\
\text { Cochrane score: } 9 / 10\end{array}$ & $\begin{array}{l}41 \text { patients with } \\
\text { cervical pain after } \\
\text { whiplash and with } \\
\text { relief of pain after } \\
\text { controlled diagnostic } \\
\text { blocks randomized to } \\
\text { intraarticular steroid } \\
\text { or local anesthetic } \\
\text { cervical facet joint } \\
\text { injections. }\end{array}$ & $\begin{array}{l}\text { Patients randomized } \\
\text { to intraarticular } \\
\text { injection of } 5.7 \mathrm{mg} \\
\text { betamethasone or } \\
1 \mathrm{ml} \text { intraarticular } \\
\text { bupivacaine. }\end{array}$ & $\begin{array}{l}\text { Return of } \\
\text { pain to } 50 \% \text { of } \\
\text { preinjection pain } \\
\text { level. }\end{array}$ & $\begin{array}{l}\text { No significant difference } \\
\text { in duration of pain relief. } \\
\text { Median duration of time } \\
\text { to return of pain to } 50 \% \\
\text { was } 3 \text { days in the steroid } \\
\text { group and } 3.5 \text { days in the } \\
\text { local anesthetic group. }\end{array}$ & $\begin{array}{l}\text { Negative short- } \\
\text { term and long- } \\
\text { term relief }\end{array}$ \\
\hline
\end{tabular}

tients in their study with presumed facet joint pain. They showed a prevalence of lumbar facet joint pain of $58 \%$ in patients with spine pain, based on inclusion criteria in Phase I of the study. Failure to exclude placebo responders may have diluted the findings of true responses, making detection of differences between the study and control groups difficult. Further, even though results were judged to be positive at 6 months in the methylprednisolone group, they performed various types of analyses and finally concluded that there was no significant difference between groups.

Fuchs et al (100) investigated the efficacy and safety of intraarticular sodium hyaluronate $(\mathrm{SH})$ compared with intraarticular glucocorticoids (triamcinolone acetonide; TA) in the treatment of chronic nonradicular lumbar pain. They included 60 patients in this randomized, controlled, blind-observer clinical study and randomly assigned to 2 groups to receive $10 \mathrm{mg}$ of $\mathrm{SH}$ or $10 \mathrm{mg}$ TA per facet joint. The facet joints on both sides at levels $L 3 / 4, L 4 / 5$, and L5/S1 were treated once 
per week under computed tomographic guidance for a total of 6 injections. Outcome parameters included a visual analog scale, Roland Morris questionnaire, Oswestry Disability questionnaire, the Low Back Outcome Score, and the Short Form 36 questionnaire. The results showed significant pain relief and improved function and quality of life with both treatments. However, this study also showed that intraarticular hyaluronate was equivalent to intraarticular steroid. The follow-up was carried out at 3 and 6 months after completion of treatment; thus, this was considered a positive study for both hyaluronic acid and steroid injections. The drawbacks of this study include lack of diagnosis of facet joint pain by controlled local anesthetic blocks which may have increased the probability of inclusion of patients without facet joint pain.

Barnsley et al (108) performed a well-conducted study, but faced criticism. They included a total of 41 patients whose origin of neck pain was posttraumatic, following whiplash. Consequently, results may not be extrapolated to treatment of patients with cervical facet joint pain from nontraumatic causes, because responses to intraarticular steroid injections is not known for cervical facet joint pain of spontaneous origin.

Kim et al (103) was included as a new prospective observational study. They classified patients with zygapophysial joint pain of $\mathrm{C} 5 / 6$ and $\mathrm{C} 6 / 7$ and treated with therapeutic cervical facet joint injections with a mixture of $0.5 \mathrm{~mL}$ of $1 \%$ lidocaine with $5 \mathrm{mg}$ of triamcinolone under fluoroscopy. They classified patients into a herniated nucleus pulposus group, myofascial pain syndrome group, and whiplash-associated disorders group. The therapeutic effects included reduction of numeric rating scale (NRS) of pain before and immediately after blockade and symptom-free periods after 12 months. The NRS scores decreased immediately after the blockade and the symptom-free duration after blockade lasted longer in the herniated nucleus pulposus group (11.3 \pm 1.7 months) as compared to the myofascial pain syndrome group (3.2 \pm 0.9 months) and the whiplash-associated disorders group ( $3.0 \pm 0.8$ months). Characteristics and results of observational studies of intraarticular facet joint injections are illustrated in Table 3 along with methodological scores.

Schulte et al (114) evaluated 39 patients with lumbar facet syndrome using a standarized protocol with injection of steroid, lidocaine, and $5 \%$ phenol under fluoroscopic control, evaluated on follow-up using a specially designed questionnaire, which included McNab criteria, visual analogue scale, and pain disability index. They reported pain relief up to 6 months after treatment and the outcome was assessed excellent or good by $62 \%$ ( 24 patients) of the patients after one month, by $41 \%$ (16 patients) after 3 months, and by $36 \%$ (14 patients) after 6 months. They concluded that facet joint injection therapy using a standardized protocol is safe, effective, and easy to perform. Several disadvantages of this study included lack of appropriate diagnosis of facet joint pain by controlled local anesthetic blocks, possibly including false-positive patients, and injecting phenol with steroid. A combination of phenol and steroid has not been recommended as phenol may be injected to induce inflammation whereas prednisolone suppresses inflammation. However, the results were positive with $62 \%$ of patients reporting excellent or good pain relief after 1 month with a single block which extended to $36 \%$ of patients after 6 months.

Folman et al (115) evaluated the effectiveness of zygapophysial joint injections on chronic cervical pain. They selected 30 patients, with pain of more than 12 months duration and with no history of trauma, after confirming the diagnosis with intraarticular, fluoroscopically-guided injections of local anesthetic. The patients were subsequently treated with intraarticular depo-methylprednisolone. Pain relief was assessed by visual analog scale. The mean time for relapse of $50 \%$ of the pre-injection level of pain was $12.47 \pm 1.89$ weeks. They concluded that selective intraarticular blockade of the facet joints may be offered as an adjuvant for diagnostic and therapeutic purposes for patients with chronic pain due to facet arthrosis in the ambulatory setting. Their results were superior to the relief of 3 days reported in patients similarly treated following whiplash neck injury; however, the study failed to include comparative local anesthetic blocks, additional outcome parameters, and long-term follow-up.

Lynch and Taylor (111) reported initial pain relief in 31 of 35 patients receiving intraarticular steroids, whereas 8 of 15 patients receiving extraarticular steroids reported initial pain relief. Long-term pain relief was reported in $62 \%$ at 3 months, and $56 \%$ at 6 months. Destouet et al (110) reported significant pain relief for 1 to 3 months in $54 \%$ of the patients and 3 to 6 months in $38 \%$ of the 54 patients. Murtagh (109) reported long-term relief of up to 6 months in 54\% of the 100 patients. Among the retrospective evalu- 
ations, Lippitt (112) reported greater than $50 \%$ relief initially in $42 \%$ of patients, which declined to $14 \%$ at 6 months and $8 \%$ at 12 months in 99 patients. Lau et al (113) also reported initial relief in $56 \%$ of the patients, which declined to $44 \%$ at 3 months, and $35 \%$ at 6 to 12 months. Thus, all the observational studies showed positive results.

\section{Effectiveness}

The results of the study by Carette et al (104) were negative at 6 months, although patients who received intraarticular methylprednisolone were considerably improved compared to the control group. Notwithstanding their suggestion that concurrent treatments may have influenced the outcomes, for purposes of this systematic review, in terms of pain relief following intraarticular facet joint injections, the outcome is considered to have been positive for lumbar facet pain at 6 months. Fuchs et al (100) showed significant pain relief, improved function, and improved quality of life with intraarticular sodium hyaluronidase and intraarticular gluococorticoids at 3 and 6 months after injections. Barnsley et al (108) reported negative results with cervical intraarticular injections of steroids and local anesthetic.

Among the nonrandomized trials, 6 prospective evaluations $(103,109-111,114,115)$ and 2 retrospective evaluations $(112,113)$ met inclusion criteria. Among the prospective trials included in the evidence synthesis, Kim et al (103) reported positive short-term and long-term relief with cervical intraarticular injections performed under fluoroscopy. Folman et al (115) also evaluated the effectiveness of facet joint injections on chronic neck pain of nontraumatic origin and noted positive short-term and negative long-term results. Schulte et al (114) reported positive results with intraarticular injections of steroid, lidocaine, and $5 \%$ phenol under fluoroscopic control. Lynch and Taylor (111) reported initial pain relief in 31 of 35 patients receiving intraarticular steroids, whereas 8 of the 15 patients receiving extraarticular steroids reported pain relief. Long-term relief was reported in $62 \%$ at 3 months and $56 \%$ at 6 months. Destouet et al (110) reported significant pain relief for 1 to 3 months in $54 \%$ of the patients and for 3 to 6 months in $38 \%$ of the patients. Murtagh (109) reported long-term relief of longer than 3 months in $54 \%$ of patients, although a large fraction of the patients included had nonspecific back pain or radicular symptom. Among the retrospective evaluations, Lippitt (112) reported greater than $50 \%$ relief initially in $42 \%$ of patients, which declined to $14 \%$ at 6 months and $8 \%$ at 12 months. Lau et al (113) reported initial relief in $56 \%$ of patients, which declined to $44 \%$ at 3 months, and $35 \%$ at 6 to 12 months.

\section{Cost Effectiveness}

No studies were performed evaluating cost effectiveness of therapeutic intraarticular facet joint injections.

\section{Level of Evidence}

For intraarticular injections of local anesthetics and steroids, there is moderate evidence for shortand long-term improvement in low back pain. The evidence is limited for short- and long-term improvement in neck pain.

\section{Medial Branch Blocks}

Our search strategy ([facet or zygapophysial] AND injection AND nerve blocks) identified 27 new references, of which 3 evaluated therapeutic medial branch blocks; 2 randomized trials $(116,117)$, and a prospective study (118). The previous systematic review identified 6 relevant articles, 4 of which were randomized clinical trials $(106,107,119,120)$ and 2 nonrandomized clinical trials $(121,122)$.

\section{Methodologic Quality}

Three new studies (116-118) met criteria for inclusion in the current analysis. In addition, 2 studies $(119,121)$ that met inclusion criteria in the previous systematic review are included in the current evidence synthesis.

Overall, 5 randomized studies met inclusion criteria $(106,107,116,117,119)$. However, Marks et al (106) and Nash (107) were excluded as they compared the effectiveness of intraarticular injections with medial branch blocks on a short-term basis with no controlled local anesthetic blocks, and with lack of longterm follow-up and outcomes. Other studies excluded were a study by Manchikanti et al (122) examining the diagnostic validity of lumbar facet joint pain by controlled, comparative local anesthetic blocks, and a second study by Manchikanti et al (120) evaluating the effect of Sarapin.

Three randomized trials were included in the evidence synthesis $(116,117,119)$. In addition, both prospective evaluations $(118,121)$ were included. Table 4 illustrates characteristics of published reports of cervical, thoracic and lumbar medial branch blocks included in the evidence synthesis. 
Table 3. Characteristics and results of observational studies of cervical and lumbar facet joint injections.

\begin{tabular}{|c|c|c|c|c|c|}
\hline Study/Methods & Participants & Intervention(s) & Outcome(s) & Result(s) & $\begin{array}{c}\text { Conclusion(s) } \\
\text { Short-term relief } \\
<6 \mathrm{wks} \\
\text { Long-term relief } \\
\geq 6 \mathrm{wks}\end{array}$ \\
\hline \multicolumn{6}{|l|}{ Cervical } \\
\hline $\begin{array}{l}\text { Kim et al (103) } \\
\begin{array}{l}\text { Prospective } \\
\text { evaluation }\end{array} \\
\text { AHRQ score: } 4 / 8\end{array}$ & $\begin{array}{l}60 \text { patients were } \\
\text { treated with } \\
\text { therapeutic cervical } \\
\text { zygapophysial joint } \\
\text { injections under } \\
\text { fluoroscopy. Patients } \\
\text { were divided into } 3 \\
\text { groups: herniated } \\
\text { nucleus pulposus } \\
\text { group, myofascial } \\
\text { pain syndrome } \\
\text { group, and whiplash- } \\
\text { associated disorders } \\
\text { group. }\end{array}$ & $\begin{array}{l}\text { Intraarticular } \\
\text { injection of } 1 \% \\
\text { lidocaine with } 5 \mathrm{mg} \\
\text { of triamcinolone } \\
\text { under fluoroscopy } \\
\text { into C5/6 and C6/7 } \\
\text { joints. }\end{array}$ & $\begin{array}{l}\text { Pain relief and } \\
\text { duration of } \\
\text { symptom-free } \\
\text { period }\end{array}$ & $\begin{array}{l}\text { The symptom-free } \\
\text { duration after blockade } \\
\text { lasted longer in the } \\
\text { herniated nucleus } \\
\text { pulposus group with } \\
11.3 \pm 1.7 \text { months than } \\
\text { the other } 2 \text { groups. In } \\
\text { the myofascial pain } \\
\text { syndrome group, relief } \\
\text { was } 3.2 \pm 0.9 \text { months } \\
\text { and in whiplash- } \\
\text { associated disorders } \\
\text { group, the relief was } 3.0 \\
\pm 0.8 \text { months. }\end{array}$ & $\begin{array}{l}\text { Positive short-term } \\
\text { and long-term relief }\end{array}$ \\
\hline $\begin{array}{l}\text { Folman et al } \\
\text { (115) } \\
\text { Prospective } \\
\text { evaluation } \\
\text { AHRQ score: } 4 / 8\end{array}$ & $\begin{array}{l}30 \text { patients, with } \\
\text { pain of more than } \\
12 \text { months duration } \\
\text { and with no history } \\
\text { of trauma, with facet } \\
\text { joint pain diagnosed } \\
\text { by intraarticular } \\
\text { fluoroscopically- } \\
\text { guided infiltration of } \\
\text { anesthetic. }\end{array}$ & $\begin{array}{l}\text { Following the } \\
\text { diagnosis of facet } \\
\text { joint pain with } \\
\text { intraarticular, } \\
\text { fluoroscopically- } \\
\text { guided local } \\
\text { anesthetic } \\
\text { injections, treated } \\
\text { with intraarticular } \\
\text { corticosteroids depo- } \\
\text { medrol } 40 \mathrm{mg} \text {. }\end{array}$ & $\begin{array}{l}\text { Pain relief } \\
\text { assessed by visual } \\
\text { analog scale. }\end{array}$ & $\begin{array}{l}\text { The mean time for } \\
\text { relapse of } 50 \% \text { of the } \\
\text { preinjection level of } \\
\text { pain was } 12.47+1.89 \\
\text { weeks. }\end{array}$ & $\begin{array}{l}\text { Positive short-term } \\
\text { and long-term relief. }\end{array}$ \\
\hline \multicolumn{6}{|l|}{ Lumbar } \\
\hline $\begin{array}{l}\text { Schulte et al } \\
(114) \\
\begin{array}{l}\text { Prospective } \\
\text { evaluation }\end{array} \\
\text { AHRQ score: } 6 / 8\end{array}$ & $\begin{array}{l}39 \text { patients with } \\
\text { chronic low back } \\
\text { pain diagnosed } \\
\text { as lumbar facet } \\
\text { syndrome. }\end{array}$ & $\begin{array}{l}\text { Injection of steroid, } \\
\text { lidocaine, and } \\
5 \% \text { phenol under } \\
\text { fluoroscopic control. }\end{array}$ & $\begin{array}{l}\text { Outcomes were } \\
\text { determined } \\
\text { on a follow-up } \\
\text { based specialty } \\
\text { questionnaire } \\
\text { including: } \\
\text { visual analog } \\
\text { scale, McNab } \\
\text { criteria, and pain } \\
\text { disability index. }\end{array}$ & $\begin{array}{l}\text { Pain relief of up to } 6 \\
\text { months was reported } \\
\text { after the treatment. The } \\
\text { outcome was assessed } \\
\text { as excellent or good by } \\
62 \% \text { of the patients after } \\
1 \text { month, by } 41 \text { after } 3 \\
\text { months, and } 36 \text { after } 6 \\
\text { months. }\end{array}$ & $\begin{array}{l}\text { Positive short-term } \\
\text { and negative long- } \\
\text { term relief }\end{array}$ \\
\hline $\begin{array}{l}\text { Murtagh (109) } \\
\text { Prospective study } \\
\text { AHRQ score: } 6 / 8\end{array}$ & $\begin{array}{l}100 \text { patients with low } \\
\text { back pain with pain } \\
\text { relief after lumbar } \\
\text { intraarticular facet } \\
\text { joint injections using } \\
\text { fluoroscopy or CT. }\end{array}$ & $\begin{array}{l}\text { Patients with } \\
\text { immediate relief } \\
\text { of pain after local } \\
\text { anesthetic injections } \\
\text { received repeat } \\
\text { intraarticular } \\
\text { injection of } 6 \mathrm{mg} \\
\text { betamethasone. }\end{array}$ & $\begin{array}{l}\text { Follow-up in } \\
\text { all patients. } \\
\text { Subjective relief } \\
\text { as determined by } \\
\text { patient response. }\end{array}$ & $\begin{array}{l}54 \% \text { of patients had } \\
\text { more than } 3 \text { months of } \\
\text { pain relief. }\end{array}$ & $\begin{array}{l}\text { Positive short-term } \\
\text { and long-term relief }\end{array}$ \\
\hline $\begin{array}{l}\text { Destouet et al } \\
(110) \\
\text { Prospective study } \\
\text { AHRQ score: } 6 / 8\end{array}$ & $\begin{array}{l}54 \text { patients studied; } \\
13 \text { had previous } \\
\text { lumbar surgery. } \\
\text { Patients received } \\
\text { intraarticular facet } \\
\text { injections. }\end{array}$ & $\begin{array}{l}1 \mathrm{ml} 0.25 \% \\
\text { bupivacaine and } \\
40 \mathrm{mg} \text { depot } \\
\text { methylprednisolone. }\end{array}$ & Pain relief. & $\begin{array}{l}54 \% \text { of patients had } \\
\text { initial relief; whereas } \\
38 \% \text { had continued } \\
\text { pain relief for } 3 \text { months } \\
\text { or longer. }\end{array}$ & $\begin{array}{l}\text { Positive short-term } \\
\text { and negative long- } \\
\text { term relief }\end{array}$ \\
\hline
\end{tabular}


Table 3 Continued. Characteristics and results of observational studies of cervical and lumbar facet joint injections.

\begin{tabular}{|c|c|c|c|c|c|}
\hline Study/Methods & Participants & Intervention(s) & Outcome(s) & Result(s) & $\begin{array}{c}\text { Conclusion(s) } \\
\text { Short-term relief } \\
<6 \text { wks } \\
\text { Long-term relief } \\
\geq 6 \text { wks }\end{array}$ \\
\hline $\begin{array}{l}\text { Lynch and Taylor } \\
\text { (111) } \\
\text { Prospective study } \\
\text { AHRQ score: } 6 / 8\end{array}$ & $\begin{array}{l}35 \text { patients in } \\
\text { intraarticular and } \\
15 \text { in extraarticular } \\
\text { group, all with } \\
\text { presumed lumbar } \\
\text { facet pain, were } \\
\text { studied. }\end{array}$ & $\begin{array}{l}\text { Intraarticular or } \\
\text { extraarticular } \\
\text { injections with } 60 \mathrm{mg} \\
\text { methylprednisolone. }\end{array}$ & Pain relief. & $\begin{array}{l}89 \% \text { of patients had } \\
\text { pain relief initially in } \\
\text { the intraarticular group; } \\
\text { intraarticular injections } \\
\text { were better than } \\
\text { extraarticular. }\end{array}$ & $\begin{array}{l}\text { Positive short-term } \\
\text { and long-term relief }\end{array}$ \\
\hline $\begin{array}{l}\text { Lippitt (112) } \\
\begin{array}{l}\text { Retrospective } \\
\text { review }\end{array} \\
\text { AHRQ score: } 5 / 8\end{array}$ & $\begin{array}{l}99 \text { patients with } \\
\text { clinical diagnosis } \\
\text { of lumbar facet } \\
\text { pain received facet } \\
\text { joint injections with } \\
\text { fluoroscopy. }\end{array}$ & $\begin{array}{l}\text { Intraarticular } \\
\text { injection of } 1 \\
\text { ml } 1 \% \text { lidocaine } \\
\text { and } 80 \mathrm{mg} \text { depot } \\
\text { methylprednisolone. }\end{array}$ & $\begin{array}{l}\text { Pain relief, return } \\
\text { to work. }\end{array}$ & $\begin{array}{l}42 \% \text { of patients had } \\
\text { initial relief which } \\
\text { declined to } 14 \% \text { at } 6 \\
\text { months. }\end{array}$ & $\begin{array}{l}\text { Negative short-term } \\
\text { and long-term relief }\end{array}$ \\
\hline $\begin{array}{l}\text { Lau et al (113) } \\
\begin{array}{l}\text { Retrospective } \\
\text { study }\end{array} \\
\text { AHRQ score: } 6 / 8\end{array}$ & $\begin{array}{l}34 \text { consecutive } \\
\text { patients with clinical } \\
\text { diagnosis of lumbar } \\
\text { facet pain received } \\
\text { facet joint injections } \\
\text { with fluoroscopy. }\end{array}$ & $\begin{array}{l}\text { Intraarticular } \\
\text { injection of } \\
\text { bupivacaine } \\
\text { and depot } \\
\text { methylprednisolone. }\end{array}$ & Pain relief. & $\begin{array}{l}56 \% \text { of patients } \\
\text { reported immediate } \\
\text { pain relief, which } \\
\text { declined to } 44 \% \text { at } 3 \\
\text { months and } 35 \% \text { at } 6 \text { to } \\
12 \text { months. }\end{array}$ & $\begin{array}{l}\text { Positive short-term } \\
\text { and negative long- } \\
\text { term relief }\end{array}$ \\
\hline
\end{tabular}

\section{Study Characteristics and Effectiveness}

Three new studies evaluated the effectiveness of medial branch blocks for managing chronic spinal pain of facet origin. Manchikanti et al (116) evaluated pain relief with cervical medial branch blocks in a randomized, double-blind, controlled trial of patients with chronic neck pain. In their preliminary report, the authors describe 60 patients with cervical facet pain diagnosed by comparative, controlled diagnostic cervical facet medial branch nerve blocks with bupivacaine, with or without steroid (betamethasone). Significant pain relief (> 50\%) and improvement in functional status were observed at 3 , 6 , and 12 months in $80 \%-87 \%, 80 \%-93 \%$, and $87 \%$ $93 \%$ of patients, respectively. The average number of treatments per patient for 1 year was about 3.5, with no difference between the groups. Duration of relief was approximately 3.5 months per treatment. Thus, satisfactory relief of chronic spinal pain of facet origin could be maintained over the course of a year with medial branch blocks repeated at clinically indicated intervals. Inclusion of steroid in the injectate did not appear to provide additional benefit. Although this was a small, randomized study, it provides further evidence that cervical facet joint medial branch blocks may offer durable relief of facet pain without neurolysis.

A second preliminary report by Manchikanti et al (117) is in press and was obtained for the current review. The study is a randomized, double-blind, controlled trial similar in design to the study described above (116). Sixty patients with lumbar facet pain diagnosed with comparative local anesthetic medial branch blocks were randomized to receive therapeutic local anesthetic medial branch blocks of lumbar facets, with or with steroid (betamethasone). Results were similar to those described for cervical facet pain. Significant pain relief and functional status improvement were observed at 3 months, 6 months, and 12 months, compared to baseline measurements, in $73 \%-87 \%, 87 \%-93 \%$, and $73 \%-93 \%$ of patients, respectively. The average number of treatments per patient for 1 year was approximately 3.5, with no significant differences noted between groups. Duration of average pain relief with each procedure was about 3.5 months. This small, randomized study provides additional evidence that medial branch blocks may be considered therapeutic for patients with chronic low back pain of facet origin. 
Table 4. Characteristics of published reports of cervical, thoracic, and lumbar medial branch blocks.

\begin{tabular}{|c|c|c|c|c|c|}
\hline Study/Methods & Participants & Intervention(s) & Outcome(s) & Result(s) & $\begin{array}{c}\text { Conclusion(s) } \\
\text { Short-term } \\
\text { relief }<3 \mathrm{mos} \\
\text { Long-term } \\
\text { relief } \geq 3 \mathrm{mos}\end{array}$ \\
\hline \multicolumn{6}{|l|}{ CERVICAL } \\
\hline $\begin{array}{l}\text { Manchikanti et al (116) } \\
\text { Cervical medial branch } \\
\text { nerve blocks } \\
\text { Randomized, double- } \\
\text { blind controlled } \\
\text { AHRQ score: } 7 / 10 \\
\text { Cochrane score: } 10 / 10\end{array}$ & $\begin{array}{l}60 \text { patients, all } \\
\text { meeting criteria } \\
\text { for cervical facet } \\
\text { joint pain. } \\
4 \text { treatment } \\
\text { groups, } 15 \\
\text { patients each. }\end{array}$ & $\begin{array}{l}\text { Cervical medial branch } \\
\text { nerve blocks in all } 4 \\
\text { treatment groups: Group } \\
\text { I serving as control with } \\
\text { bupivacaine only; Group II } \\
\text { with bupivacaine+sarapin; } \\
\text { Group III with bupivacaine } \\
\text { +betamethasone; Group IV } \\
\text { with bupivacaine+sarapin+ } \\
\text { betamethasone. }\end{array}$ & $\begin{array}{l}\text { Measured numeric } \\
\text { pain scores, } \\
\text { Neck Pain index, } \\
\text { opioid intake, and } \\
\text { employment status } \\
\text { at baseline, } 3,6 \text {, } \\
\text { and } 12 \text { mos. }\end{array}$ & $\begin{array}{l}\text { Significant pain relief } \\
(\geq 50 \%) \text { and functional } \\
\text { status improved at } 3,6 \text {, } \\
\text { and } 12 \text { mos. No significant } \\
\text { difference in the duration } \\
\text { of average pain relief } \\
\text { among the groups. } 80 \%- \\
93 \% \text { of patients reported } \\
\text { significant relief at } 3,6 \text { and } \\
12 \text { mos. }\end{array}$ & $\begin{array}{l}\text { Positive short- } \\
\text { term and long- } \\
\text { term relief }\end{array}$ \\
\hline $\begin{array}{l}\text { Manchikanti et al (121) } \\
\text { Cervical medial branch } \\
\text { blocks } \\
\text { Prospective } \\
\text { AHRQ score: } 8 / 8\end{array}$ & $\begin{array}{l}100 \text { consecutive } \\
\text { patients with } \\
\text { cervical facet } \\
\text { pain diagnosed } \\
\text { by comparative, } \\
\text { controlled local } \\
\text { anesthetic blocks. }\end{array}$ & $\begin{array}{l}\text { Medial branch blocks } \\
\text { with fluoroscopy } \\
\text { with bupivacaine } \\
\text { with or without } \\
\text { methylprednisolone. } \\
\text { Patients had repeat blocks } \\
\text { as clinically indicated. }\end{array}$ & $\begin{array}{l}\text { Pain relief } \\
\text { Oswestry } \\
\text { Disability Index, } \\
\text { psychological } \\
\text { status, work status } \\
\text { Timings: } 3 \text { mos, } 6 \\
\text { mos, and } 12 \text { mos. }\end{array}$ & $\begin{array}{l}\text { Significant pain relief at } 3 \text {, } \\
6 \text {, and } 12 \text { mos, compared } \\
\text { to baseline measurements. } \\
\text { There was also significant } \\
\text { improvement in disability } \\
\text { status, psychological status, } \\
\text { and return to work. Significant } \\
\text { pain relief was observed at } \\
92 \% \text { at } 3 \text { mos, } 82 \% \text { at } \\
6 \text { mos, and } 56 \% \text { at } 12 \text { mos. }\end{array}$ & $\begin{array}{l}\text { Positive short- } \\
\text { term and long- } \\
\text { term relief }\end{array}$ \\
\hline \multicolumn{6}{|l|}{ THORACIC } \\
\hline $\begin{array}{l}\text { Manchikanti et al (118) } \\
\text { Thoracic medial branch } \\
\text { nerve blocks } \\
\text { Prospective outcome } \\
\text { study } \\
\text { AHRQ score: } 8 / 10\end{array}$ & $\begin{array}{l}55 \text { consecutive } \\
\text { patients, } \\
\text { all meeting } \\
\text { diagnostic } \\
\text { criteria for } \\
\text { thoracic facet } \\
\text { joint pain. }\end{array}$ & $\begin{array}{l}\text { Thoracic facet joint } \\
\text { nerve blocks performed } \\
\text { using bupivacaine with } \\
\text { or without Sarapin and } \\
\text { depomethylprednisolone. }\end{array}$ & $\begin{array}{l}\text { Measured numeric } \\
\text { pain scores, } \\
\text { Oswestry Disability } \\
\text { Index, employment } \\
\text { status, and Pain } \\
\text { Patient Profile at 3, 6, } \\
\text { 12, 24, and } 36 \text { mos. }\end{array}$ & $\begin{array}{l}\text { Significant ( } \geq 50 \% \text { ), was } \\
\text { observed in } 71 \% \text { of the } \\
\text { patients at } 3 \text { mos and } 6 \\
\text { mos, } 76 \% \text { at } 12 \text { mos, } 71 \% \text { at } \\
24 \text { mos, and } 69 \% \text { at } 36 \text { mos. }\end{array}$ & $\begin{array}{l}\text { Positive short- } \\
\text { term and long- } \\
\text { term relief }\end{array}$ \\
\hline \multicolumn{6}{|l|}{ LUMBAR } \\
\hline $\begin{array}{l}\text { Manchikanti et al (117) } \\
\text { Lumbar facet joint } \\
\text { nerve blocks } \\
\text { Prospective, } \\
\text { randomized, double- } \\
\text { blind controlled } \\
\text { AHRO score: } 8 / 10 \\
\text { Cochrane score: } 8 / 10 \\
\end{array}$ & $\begin{array}{l}60 \text { patients, } \\
\text { all meeting } \\
\text { diagnostic } \\
\text { criteria for } \\
\text { lumbar facet } \\
\text { joint pain. } \\
4 \text { treatment } \\
\text { groups, } 15 \\
\text { patients each. }\end{array}$ & $\begin{array}{l}\text { Lumbar facet joint nerve } \\
\text { block injections in all } 4 \\
\text { treatment groups: } \\
\text { Group I serving as } \\
\text { control with bupivacaine } \\
\text { only; Group II with } \\
\text { bupivacaine+sarapin; Group } \\
\text { III with bupivacaine+steroid; } \\
\text { Group IV with bupivacaine+ } \\
\text { sarapin+steroid } \\
\end{array}$ & $\begin{array}{l}\text { Measured } \\
\text { numeric pain } \\
\text { scores, Oswestry } \\
\text { Disability Index, } \\
\text { opioid intake, and } \\
\text { employment status } \\
\text { at baseline, } 3,6 \text {, } \\
\text { and } 12 \text { mos. }\end{array}$ & $\begin{array}{l}\text { Significant pain relief } \\
(\geq 50 \%) \text { and functional } \\
\text { status improved at } 3,6 \text {, } \\
\text { and } 12 \text { mos. No significant } \\
\text { differences noted between } \\
\text { steroid and nonsteroid } \\
\text { treatment groups. } 73 \% \text { - } \\
93 \% \text { of patients reported } \\
\text { significant relief at } 3,6 \text {, and } \\
12 \text { mos. }\end{array}$ & $\begin{array}{l}\text { Positive short- } \\
\text { term and long- } \\
\text { term relief }\end{array}$ \\
\hline $\begin{array}{l}\text { Manchikanti et al (119) } \\
\text { Lumbar medial branch } \\
\text { blocks } \\
\text { Randomized, controlled } \\
\text { trial } \\
\text { AHRQ score: } 8 / 10 \\
\text { Cochrane score: } 6 / 10\end{array}$ & $\begin{array}{l}200 \text { patients with } \\
\text { low back pain } \\
\text { were evaluated. } \\
73 \text { patients } \\
\text { were enrolled } \\
\text { in study after } \\
\text { confirmation } \\
\text { of facet pain } \\
\text { by controlled } \\
\text { diagnostic facet } \\
\text { nerve blocks. }\end{array}$ & $\begin{array}{l}\text { Medial branch blocks } \\
\text { with fluoroscopy. Patients } \\
\text { randomized into } 2 \\
\text { groups: local anesthetic, } \\
\text { bupivacaine, with or } \\
\text { without Sarapin }{ }^{\circ} \text { Patients } \\
\text { had repeat procedures as } \\
\text { clinically indicated. }\end{array}$ & $\begin{array}{l}\text { Outcomes were } \\
\text { evaluated over a } \\
\text { period of } 2 \frac{1 / 2}{2} \text { years. } \\
\text { Measurements } \\
\text { were performed } \\
\text { at } 1,3,6,12,18, \\
24 . \text { and } 32 \text { mos. } \\
\text { Outcomes included } \\
\text { pain relief, } \\
\text { physical health, } \\
\text { psychological } \\
\text { status, narcotic } \\
\text { intake, and } \\
\text { employment status. }\end{array}$ & $\begin{array}{l}\text { Cumulative significant relief } \\
\text { with } 1 \text { to } 3 \text { injections up to } \\
2.5 \text { years. Overall, significant } \\
\text { relief for a mean of } 6.5 \text { mos. } \\
\text { Significant improvement } \\
\text { was noted in overall health } \\
\text { status with improvement } \\
\text { in pain relief, psychological } \\
\text { status, and return to work } \\
\text { status. Significant relief was } \\
\text { seen with } 1 \text { to } 3 \text { injections in } \\
100 \% \text { of patients at } 3 \text { mos, } \\
75 \%-88 \% \text { at } 6 \text { mos, and } 17 \%- \\
25 \% \text { at } 12 \text { mos. }\end{array}$ & $\begin{array}{l}\text { Positive short- } \\
\text { term and long- } \\
\text { term relief }\end{array}$ \\
\hline
\end{tabular}


An observational study by Manchikanti and colleagues (118) examined the therapeutic benefit of thoracic medial branch blocks in a prospective outcome study. In 55 consecutive patients with thoracic facet pain confirmed by comparative diagnostic facet nerve blocks, more than two-thirds of patients obtained significant pain relief $(>50 \%)$ with bupivacaine and methylprednisolone compared to baseline measurements, $(71 \%$ of the patients at 3 months and 6 months, $76 \%$ of the patients at 12 months, $71 \%$ at 24 months, and $69 \%$ at 36 months). Patients received approximately 3 to 4 blocks per year with an average duration of relief per treatment of about 4 months. As with the cervical and lumbar facet nerve block studies (vide supra), this article provides evidence that repetitive medial branch blocks may provide durable relief of spinal pain of thoracic facet joint origin.

Manchikanti et al (119) evaluated 73 patients positive for lumbar facet joint pain by means of controlled, comparative local anesthetic blocks. They randomly allocated patients into 2 groups, either therapeutic medial branch blocks with a local anesthetic and Sarapin or a mixture of local anesthetic, Sarapin, and methylprednisolone. Significant improvement was documented in both groups in various parameters of pain relief, functional status, opioid intake, return to work, and psychological status. Significant cummulative pain relief was seen with 1 to 3 injections in $100 \%$ of the patients up to 1 to 3 months, $82 \%$ of the patients for 4 to 6 months, and $21 \%$ for 7 to 12 months. The mean relief was $6.5 \pm 0.76$ months.

Manchikanti et al (121) evaluated the therapeutic effectiveness of cervical facet joint nerve blocks in chronic neck pain in a prospective outcome study. They evaluated 100 consecutive patients meeting the diagnostic criteria of facet joint pain by means of comparative, controlled diagnostic blocks. There were significant differences in numeric pain scores and pain relief (> 50\%) at 3 months (92\%), 6 months $(82 \%)$, and 12 months (56\%) compared to baseline measurements. There was significant improvement in functional status, psychological status, and employment among patients eligible for employment (employed and unemployed) from baseline to 12 months.

Manchikanti et al (118) in a prospective outcome study with minimum of 1-year follow-up, evaluated the therapeutic role of thoracic medial branch blocks in managing chronic thoracic pain. Fifty-five consecutive patients meeting the diagnostic criteria of thoracic facet joint pain by means of comparative, controlled diagnostic blocks were included. Medial branch blocks were performed with local anesthetic, with or without steroids. The results showed significant differences in numeric pain scores and significant pain relief $(50 \%$ or $>$ ) in $71 \%$ of the patient at 3 months and 6 months, $76 \%$ at 12 months, $71 \%$ at 24 months, and $69 \%$ at 36 months, compared to baseline measurements. Functional improvement was demonstrated at 1 year, 2 years, and 3 years from baseline. There was significant improvement with increase in employment among the patients eligible for employment from baseline to 1 year, 2 years, and 3 years in conjunction with improved psychological function.

\section{Cost Effectiveness}

The cost effectiveness of lumbar facet joint nerve blocks was evaluated by Manchikanti et al (119) with 1 -year improvement of quality of life at $\$ 3,461$.

\section{Level of Evidence}

Evidence for therapeutic medial branch blocks in the lumbar, cervical, and thoracic spine is moderate for short- and long-term pain relief.

\section{Medial Branch Neurotomy}

Percutaneous neurotomy of medial branches is a procedure that offers pain relief by denervation of the nerves that innervate a painful joint. The denervation may be performed by radiofrequency thermoneurolysis utilizing thermal or pulsed mode, cryoneurolysis, or laser denervation.

For the current systematic review, a randomized trial by van Wijk and colleagues (123) that evaluated the effectiveness of radiofrequency neurotomy for relief of lumbar facet joint pain. The study was of high quality, based on AHRQ and Cochrane criteria, and assessed the effectiveness of radiofrequency neurolysis on lumbar facet pain in 81 patients randomized to active radiofrequency or sham lesioning. The combined outcome measure, based on the authors' multidimensional outcome tool (visual analogue scale back pain measurements, changes in daily physical activities, and analgesic use) showed no significant difference between radiofrequency denervation and sham. However, the global perceived effect, a secondary outcome, was better in the radiofrequency group. In selected patients, radiofrequency may have been better than sham intervention. Unfortunately, the study by van Wijk (123) failed to meet inclusion criteria and was subject to criticism on technical grounds. Bogduk (124) noted that the radiofrequency needle position 
was improper. Needle tips were positioned perpendicular and too far lateral to the medial branch nerves to ensure denervation; this could increase the likelihood of treatment failure. It should also be noted that comparative, controlled diagnostic blocks were not used, which may have increased the false-positive rate of facet pain diagnosis, reducing the likelihood of noting a statistical effect with active treatment. Further, the relief at which they judged a patient to be positive for facet joint pain was only $50 \%$.

Haspeslagh et al (125), in a pseudo-randomized controlled trial of cervical radiofrequency lesions as a treatment for cervicogenic headache, evaluated 30 patients according to Sjaastad diagnostic criteria, with 15 patients receiving a sequence of radiofrequency treatments with cervical facet joint denervation followed by cervical dorsal root ganglion lesions when necessary, and another 15 patients undergoing local injections with steroid and anesthetic at the greater occipital nerve, followed by transcutaneous electrical nerve stimulation when necessary. There were no statistically significant differences between the 2 treatment groups at any time point in the trial. They concluded that they did not find evidence that radiofrequency treatment of cervical facet joints and upper dorsal root ganglia is a better treatment than infiltration of the greater occipital nerve, followed by TENS for patients fulfilling the clinical criteria of cervicogenic headache. This study was problematic, not only in the diagnosis but also in the application of technique. The authors claim that they developed a sequence of various cervical radiofrequency neurotomies that proved successful in a prospective pilot trial with 15 chronic headache patients. Their diagnosis was not established by controlled diagnostic blocks; and the treatments targeted toward different structures: cervical facet joints and dorsal root ganglion compared to occipital nerves. Thus, this study was excluded from evidence synthesis.

Numerous prospective studies were identified. Barnsley (126) and Shin et al (127) evaluated the effectiveness of cervical medial branch radiofrequency, Birkenmaier et al (128) and Staender et al (129) evaluated the effectiveness of cryoneurolysis in managing lumbar facet syndrome, Mogalles et al (130) evaluated percutaneous laser denervation. There was also a retrospective evaluation of facet joint pain (131). The effectiveness of pulsed radiofrequency was also evaluated in 2 studies $(132,133)$. The studies by Staender et al (129), Mogalles et al (130), Barnsley (126), Marti-
nez-Suarez et al (131), and Birkenmaier et al (128) met inclusion criteria.

However, the studies by van Wijk et al (123) and Shin et al (127) failed to meet inclusion criteria. Two studies evaluating pulsed radiofrequency $(132,133)$ were also excluded.

In our previous systematic review, 2 randomized trials, one of the cervical spine (134) and 1 involving the lumbar spine (135) met inclusion criteria and are included in the present analysis. Four observational studies of cervical medial branch neurotomy (136-139) met criteria and are included from the previous review. Likewise, 6 observational studies of lumbar medial branch neurotomy (138-143) and 2 observational studies of thoracic medial branch neurotomy $(138,144)$ met inclusion criteria in the previous systematic review and are included in the present analysis.

\section{Methodological Criteria}

For the current systematic review, 8 randomized trials evaluating the efficacy of medial branch facet radiofrequency neurotomy were identified $(123,125$, $134,135,143-146), 2$ with cervical medial branch neurotomy $(125,134)$ and 6 with lumbar medial branch neurotomy $(123,135,143-146)$. Among the randomized trials, only 2 met inclusion criteria. The study by Lord et al (134) evaluated the effectiveness of percutaneous radiofrequency neurotomy from cervical zygapophysial joint pain and the study by van Kleef et al (135) evaluated the effectiveness of radiofrequency lumbar facet denervation for chronic low back pain. The methodological scoring for both of the studies is illustrated in Table 5, along with study descriptions.

Other randomized trials were excluded as they failed to meet inclusion or methodological criteria. The study by LeClaire et al (144), which appeared to be a well-performed, double-blind, placebo-controlled trial, had several deficiencies. The study failed to define the study population using appropriate diagnostic criteria. This was considered a major error, because patients were evaluated with a single diagnostic block and pain relief was considered to be more than one day's relief during the 7-day period following the diagnostic block. Consequently, any results or conclusions also could be erroneous. A study by Gallagher et al (143) was not included because it used the invalidated Shealy technique, failed to describe appropriate diagnostic techniques and outcome analysis, and it was not clear whether interventions were performed with fluoroscopy. Three other studies were excluded from inclusion 
and evidence synthesis; 1 study used intraarticular facet joint denervation (145), which is not medial branch neurotomy and is of unclear clinical relevance, and the other 2 studies $(147,148)$ described radiofrequency lesioning of dorsal root ganglia. The recent study by van Wijk (123) also failed to meet inclusion criteria and was excluded. This study failed to utilize comparative, controlled diagnostic blocks and reasonable pain relief criteria. In addition, needle tips were positioned perpendicular and 2 far lateral to the medial branch nerves to ensure denervation. A study by Haspeslagh et al (125) was excluded as they performed an inadequate procedure for cervical facet radiofrequency.

There were 15 observational studies (126-131, $136-142,149,150)$ identified that met inclusion criteria and were included in the review. Of these, 5 studies evaluated (Table 5) cervical facet radiofrequency thermoneurolysis $(126,136-139)$ and 11 studies evaluated (Table 6) low back pain $(127-131,138,139,140-142,149)$; 2 of the studies $(138,150)$ included evaluations of thoracic facet joint pain (Table 7 ).

Birkenmaier et al (128) and Staender et al (129) utilized cryoneurolysis for medial branch neurotomy. Both studies were included in the analysis of evidence synthesis. Mogalles et al (130) utilized percutaneous laser denervation for medial branch neurotomy.

\section{Study Characteristics}

Lord et al (134) evaluated percutaneous radiofrequency neurotomy for management of chronic cervical facet joint pain in a randomized, double-blind, placebo-controlled trial. They concluded that for patients with chronic cervical facet joint pain, confirmed by double-blinded, placebo-controlled local anesthetic blocks, percutaneous radiofrequency neurotomy with multiple lesions of target nerves can provide long-lasting relief.

van Kleef et al (135) randomized 31 patients to radiofrequency neurolysis or sham lesioning of lumbar facet medial branch nerves. At 3, 6, and 12 months, there were statistically more successes in the treatment group than the sham group. These results demonstrated that radiofrequency denervation of the lumbar facet joints can be effective for pain reduction in patients with lumbar facet joint pain.

The recent observational study by Barnsley (126) evaluated the results of radiofrequency for cervical facet joint pain as applied in usual clinical practice. All patients who underwent radiofrequency had the diagnosis of cervical facet joint pain made with com- parative local anesthetic blocks. The primary outcome was duration of complete pain relief for all consecutive procedures performed over a two-year period. Forty-seven procedures were performed on 35 patients. Twelve patients underwent 2 procedures and 2 patients were lost to follow-up. Sixty percent of patients obtained complete pain relief for a duration in excess of 12 weeks. Overall, the mean duration of complete pain relief was 35 weeks.

A prospective study by Shin et al (127) evaluated the role of radiofrequency neurotomy of cervical medial branches for chronic cervicobrachialgia, radiating in typical facet joint patterns to the neck and shoulder. Twenty-eight patients with facet joint pain were identified with comparative local anesthetic blocks and subsequently underwent radiofrequency neurolysis. The primary outcome was pain relief at 1, 2, 3, 6, 9 and 12 months. Sixty-eight percent of patients had a successful outcome ( $>50 \%$ pain relief) after 6 months of follow-up; $29 \%$ reported complete pain relief.

Birkenmaier et al (128) evaluated the benefit of cryodenervation (cryoneurolysis) for lumbar facet pain diagnosed with positive medial branch local anesthetic blocks. The study design was a prospective case series. Outcomes were low back pain improvement, improved activity, and overall satisfaction. Forty-six out of 50 patients completed the study. Outcomes were determined at 6 weeks and 3, 6, and 12 months. Overall, mean low back pain was significantly decreased from baseline at 3, 6, and 12 months. Significant improvement was seen in $72 \%$ of patients at 6 weeks, $70 \%$ at 3 months, and $57 \%$ at 12 months.

Staender et al (129) performed computerized tomography-guided cryorhizotomy in 76 patients with lumbar facet joint syndrome. In this prospective evaluation a diagnosis was established after 3 positive CT-guided medial branch nerve blocks. Outcome measurements, which included visual analog score, use of medication, ability to work, and physical condition were evaluated at 3 days, 3 months, and every 3 months thereafter, with a median follow-up period of 22.5 months, ranging from 6 to 43 months. They reported significant reductions in the VAS at 3 months and 6 months postoperatively. In $40 \%$ of patients, pain was reduced for 12 months or longer. Eighteen patients underwent a second, 7 a third, and 1 a fourth cryorhizotomy.

Mogalles et al (130) evaluated percutaneous laser denervation of the zygapophysial joints for lumbar facet pain. This prospective evaluation included per- 
Table 5. Characteristics of results of studies of cervical medial branch neurotomy.

\begin{tabular}{|c|c|c|c|c|c|}
\hline Study/Methods & Participants & Intervention(s) & Outcome(s) & Result(s) & $\begin{array}{c}\text { Conclusions } \\
\text { Short-term } \\
\text { relief }<3 \mathrm{mos} \\
\text { Long-term } \\
\text { relief } \geq 3 \mathrm{mos} .\end{array}$ \\
\hline $\begin{array}{l}\text { Lord et al (134) } \\
\text { Cervical zygapophysial } \\
\text { joint nerve } \\
\text { radiofrequency } \\
\text { Randomized, placebo- } \\
\text { controlled, double-blind } \\
\text { trial } \\
\text { AHRQ score: } 9 / 10 \\
\text { Cochrane score: } 9 / 10\end{array}$ & $\begin{array}{l}24 \text { patients } \\
\text { with neck pain } \\
>3 \text { months' } \\
\text { duration in } \\
\text { cervical spine, } \\
\text { confirmed } \\
\text { by controlled } \\
\text { blocks } \\
\text { Control=12 } \\
\text { Treatment }=12 \text {. }\end{array}$ & $\begin{array}{l}\text { RF group lesion } \\
90 \text { sec lesion at } \\
80^{\circ} \mathrm{C} \text { of medial } \\
\text { branch; Control } \\
\text { group received } \\
\text { sham treatment } \\
\text { with electrode } \\
\text { insertion. }\end{array}$ & $\begin{array}{l}3,6 \text {, and } 12 \text { month } \\
\text { follow-up; } \\
0 \text { to } 5 \text { of } 100 \text { on VAS } \\
\text { scale; Word count } \\
3 \text { or less on McGill } \\
\text { Pain questionnaire. }\end{array}$ & $\begin{array}{l}\text { Median time to return of } \\
\text { pain in treatment group } \\
\text { was } 263 \text { days; } 8 \text { days in } \\
\text { control group; } 10 \text { patients } \\
\text { underwent second } \\
\text { procedures with varying } \\
\text { results. }\end{array}$ & $\begin{array}{l}\text { Positive short- } \\
\text { term and long- } \\
\text { term relief }\end{array}$ \\
\hline $\begin{array}{l}\text { Barnsley et al (126) } \\
\text { Cervical zygapophysial } \\
\text { joint nerve } \\
\text { radiofrequency. } \\
\text { Observational study } \\
\text { AHRO score: } 7 / 8\end{array}$ & $\begin{array}{l}35 \text { consecutive } \\
\text { patients, } \\
\text { all meeting } \\
\text { diagnostic } \\
\text { criteria for } \\
\text { cervical } \\
\text { zygapophysial } \\
\text { joint pain. }\end{array}$ & $\begin{array}{l}\text { Cervical } \\
\text { zygapophysial } \\
\text { joint nerve } \\
\text { radiofrequency } \\
\text { neurotomy. }\end{array}$ & $\begin{array}{l}\text { Duration of } \\
\text { complete relief of } \\
\text { pain. }\end{array}$ & $\begin{array}{l}74 \% \text { of patients treated } \\
\text { obtained complete pain } \\
\text { relief for a mean duration } \\
\text { of } 35 \text { weeks ( } 8.7 \text { months). }\end{array}$ & $\begin{array}{l}\text { Positive short- } \\
\text { term and long- } \\
\text { term relief }\end{array}$ \\
\hline $\begin{array}{l}\text { McDonald et al (137) } \\
\text { Prospective, } \\
\text { nonrandomized } \\
\text { AHRQ score: } 7 / 8\end{array}$ & $\begin{array}{l}28 \text { patients with } \\
\text { cervical facet } \\
\text { pain diagnosed } \\
\text { by comparative } \\
\text { local anesthetic } \\
\text { blocks. }\end{array}$ & $\begin{array}{l}\text { Cervical } \\
\text { facet nerve } \\
\text { radiofrequency; } \\
\text { repeated when } \\
\text { pain returned. }\end{array}$ & $\begin{array}{l}\text { Pain relief and } \\
\text { duration of benefit. }\end{array}$ & $\begin{array}{l}\text { Complete pain relief } \\
\text { obtained in } 71 \% \text { of } \\
\text { patients after initial } \\
\text { procedure. Median } \\
\text { duration of relief was } \\
219 \text { days for all patients; } \\
422 \text { days for initial } \\
\text { responders. }\end{array}$ & $\begin{array}{l}\text { Positive short- } \\
\text { term and long- } \\
\text { term relief }\end{array}$ \\
\hline $\begin{array}{l}\text { Sapir and Gorup (136) } \\
\text { Prospective } \\
\text { nonrandomized } \\
\text { AHRQ score: } 7 / 8\end{array}$ & $\begin{array}{l}46 \text { patients with } \\
\text { cervical whiplash } \\
\text { symptoms } \\
\text { completed the } \\
\text { study. Litigant } \\
\text { and non- } \\
\text { litigant patients } \\
\text { included. }\end{array}$ & $\begin{array}{l}\text { The 2-phase } \\
\text { diagnostic } \\
\text { cervical medial } \\
\text { branch blocks, } \\
\text { followed by } \\
\text { radiofrequency } \\
\text { neurotomy. }\end{array}$ & $\begin{array}{l}\text { VAS and self-report } \\
\text { of improvement } \\
\text { (SRI). }\end{array}$ & $\begin{array}{l}\text { The difference between } \\
\text { groups in the degree } \\
\text { of symptomatology or } \\
\text { response to treatment did } \\
\text { not reach significance. }\end{array}$ & $\begin{array}{l}\text { Positive short- } \\
\text { and long-term } \\
\text { relief }\end{array}$ \\
\hline $\begin{array}{l}\text { Shin et al (127) } \\
\text { Radiofrequency } \\
\text { neurotomy of lumbar } \\
\text { medial branch nerves. } \\
\text { Observational study } \\
\text { AHRO score: } 5 / 8\end{array}$ & $\begin{array}{l}28 \text { consecutive } \\
\text { pts, all meeting } \\
\text { diagnostic } \\
\text { criteria for } \\
\text { cervical facet } \\
\text { joint pain. }\end{array}$ & $\begin{array}{l}\text { Cervical } \\
\text { medial branch } \\
\text { radiofrequency } \\
\text { neurotomy }\end{array}$ & $\begin{array}{l}\text { Measured VAS scores } \\
\text { at } 1,2,3,6,9 \text {, and } 12 \\
\text { months. Outcomes } \\
\text { graded as successful } \\
(>75 \%) ; \text { moderate } \\
(50-75 \%) \text {; and failure } \\
(<50 \%) \text { improvement }\end{array}$ & $\begin{array}{l}69 \% \text { of pts treated } \\
\text { reported successful } \\
\text { outcome after } 6 \text { months. }\end{array}$ & $\begin{array}{l}\text { Positive for } \\
\text { short-term and } \\
\text { long-term relief }\end{array}$ \\
\hline $\begin{array}{l}\text { Tzaan and Tasker (138) } \\
\text { Retrospective } \\
\text { AHRQ score: } 5 / 8\end{array}$ & $\begin{array}{l}118 \text { consecutive } \\
\text { procedures } \\
\text { in } 90 \text { patients } \\
\text { diagnosed by } \\
\text { local anesthetic } \\
\text { facet nerve block. }\end{array}$ & $\begin{array}{l}\text { Radiofrequency } \\
\text { facet neurotomy } \\
\text { at involved } \\
\text { levels. }\end{array}$ & Pain relief. & $\begin{array}{l}41 \% \text { of patients had }>50 \% \\
\text { relief at an average follow- } \\
\text { up of } 5.6 \text { months. }\end{array}$ & $\begin{array}{l}\text { Negative short- } \\
\text { term and long- } \\
\text { term relief }\end{array}$ \\
\hline $\begin{array}{l}\text { Schaerer (139) } \\
\text { Retrospective } \\
\text { AHRQ score: } 5 / 8\end{array}$ & $\begin{array}{l}117 \text { patients } \\
\text { with a total of } \\
50 \text { cervical } \\
\text { procedures. }\end{array}$ & $\begin{array}{l}\text { Radiofrequency } \\
\text { facet neurotomy } \\
\text { at involved } \\
\text { levels. }\end{array}$ & $\begin{array}{l}\text { Pain, analgesics, and } \\
\text { mood. }\end{array}$ & $\begin{array}{l}50 \% \text { of } 50 \text { patients had } \\
>50 \% \text { pain relief, with } \\
\text { average follow-up of } 13.7 \\
\text { months. }\end{array}$ & $\begin{array}{l}\text { Negative short- } \\
\text { term and long- } \\
\text { term relief }\end{array}$ \\
\hline
\end{tabular}


Table 6. Characteristics of results of studies of lumbar medial branch neurotomy.

\begin{tabular}{|c|c|c|c|c|c|}
\hline Study/Methods & Participants & Intervention(s) & Outcome(s) & Result(s) & $\begin{array}{l}\text { Conclusion(s) } \\
\text { Short-term } \\
\text { relief }<3 \mathrm{mos} \\
\text { Long-term } \\
\text { relief } \geq 3 \mathrm{mos} \\
\end{array}$ \\
\hline $\begin{array}{l}\text { van Kleef et al (135) } \\
\text { Lumbar zygapophysial } \\
\text { joint radiofrequency } \\
\text { Randomized, placebo- } \\
\text { controlled, double-blind } \\
\text { trial } \\
\text { AHRQ score: } 9 / 10 \\
\text { Cochrane score: } 7 / 10\end{array}$ & $\begin{array}{l}31 \text { pts ( } 16 \text { in } \\
\text { Control, } 15 \text { in } \\
\text { Treatment) with } \\
\text { low back pain } \\
\text { of at least } 12 \\
\text { months' duration, } \\
\text { confirmed } \\
\text { by diagnostic } \\
\text { controlled blocks. }\end{array}$ & $\begin{array}{l}\mathrm{RF} \text { group lesion } \\
60 \text { sec at } 80^{\circ} \mathrm{C} \text { of } \\
\text { medial branch; } \\
\text { Control group } \\
\text { received sham } \\
\text { treatment } \\
\text { with electrode } \\
\text { insertion. }\end{array}$ & $\begin{array}{l}2,3,6 \text {, and } 12 \\
\text { months follow- } \\
\text { up. } \\
\text { Number of } \\
\text { successes ( }>2 \mathrm{pt} \\
\text { reduction VAS, }> \\
50 \% \text { Global and } \\
\text { Oswestry). }\end{array}$ & $\begin{array}{l}\text { Statistically higher } \\
\text { improvement in } \\
\text { treatment than control } \\
\text { at the indicated times } \\
\text { (e.g.; } 67 \% \text { treat, } 37 \% \\
\text { sham at } 2 \text { months). }\end{array}$ & $\begin{array}{l}\text { Positive short- } \\
\text { term and long- } \\
\text { term relief }\end{array}$ \\
\hline $\begin{array}{l}\text { Dreyfuss et al (140) } \\
\text { Prospective } \\
\text { nonrandomized } \\
\text { AHRQ score: } 8 / 8\end{array}$ & $\begin{array}{l}15 \text { pts with } \\
\text { chronic lumbar } \\
\text { facet pain } \\
\text { diagnosed by } \\
\text { comparative local } \\
\text { anesthetic blocks. }\end{array}$ & $\begin{array}{l}\text { Lumbar } \\
\text { radiofrequency; } \\
\text { EMG of } \\
\text { multifidus muscle } \\
\text { used to determine } \\
\text { accuracy of } \\
\text { neurotomy. }\end{array}$ & $\begin{array}{l}\text { Pain relief, } \\
\text { functions, EMG } \\
\text { at } 6 \text { weeks; } \\
\text { pain relief } \\
\text { and outcomes } \\
\text { assessment at } 3,6, \\
\text { and } 12 \text { months. }\end{array}$ & $\begin{array}{l}60 \% \text { of pts obtained at } \\
\text { least } 90 \% \text { relief of pain } \\
\text { at } 12 \text { months, and } 87 \% \\
\text { at least } 60 \% \text { relief at } 12 \\
\text { months. }\end{array}$ & $\begin{array}{l}\text { Positive short- } \\
\text { term and long } \\
\text { term relief }\end{array}$ \\
\hline $\begin{array}{l}\text { Mogalles et al (130) } \\
\text { Prospective evaluation. } \\
\text { AHRQ score: } 6 / 8\end{array}$ & $\begin{array}{l}15 \text { pts were } \\
\text { included with } \\
\text { chronic low back } \\
\text { pain diagnosed } \\
\text { with facet pain } \\
\text { by means of } \\
\text { controlled, } \\
\text { comparative local } \\
\text { anesthetic blocks }\end{array}$ & $\begin{array}{l}\text { Laser denervation } \\
\text { of medial } \\
\text { branches. }\end{array}$ & $>50 \%$ pain relief. & $\begin{array}{l}8 \text { of the } 15 \text { experienced } \\
\text { complete relief and } 6 \\
\text { experienced more than } \\
50 \% \text { pain relief. }\end{array}$ & $\begin{array}{l}\text { Positive short- } \\
\text { term and long- } \\
\text { term relief }\end{array}$ \\
\hline $\begin{array}{l}\text { Vad et al (142) } \\
\text { Prospective } \\
\text { nonrandomized } \\
\text { AHRQ score: } 8 / 8\end{array}$ & $\begin{array}{l}12 \text { pts with sports- } \\
\text { related low back } \\
\text { pain diagnosed } \\
\text { with medial } \\
\text { branch blocks. }\end{array}$ & $\begin{array}{l}\text { Radiofrequency } \\
\text { neurotomy of the } \\
\text { lumbar facets. }\end{array}$ & $\begin{array}{l}\text { Pain relief } \\
\text { and return to } \\
\text { pretreatment } \\
\text { level of function. }\end{array}$ & $\begin{array}{l}\text { Mean duration of } \\
\text { pain relief: } 1.3 \text { years. } \\
83 \% \text { of pts returned to } \\
\text { pretreatment level of } \\
\text { function. }\end{array}$ & $\begin{array}{l}\text { Positive short- } \\
\text { term and long- } \\
\text { term relief }\end{array}$ \\
\hline $\begin{array}{l}\text { Birkenmaier et al (128) } \\
\text { Cryodenervation of lumbar } \\
\text { medial branch nerves } \\
\begin{array}{l}\text { Prospective clinical case } \\
\text { series }\end{array} \\
\text { AHRQ score: } 5 / 8\end{array}$ & $\begin{array}{l}46 \text { consecutive pts } \\
\text { meeting criteria } \\
\text { for refractory } \\
\text { nonsciatic low } \\
\text { back pain of at } \\
\text { least } 3 \text { months } \\
\text { with positive } \\
\text { diagnostic medial } \\
\text { branch blocks. }\end{array}$ & $\begin{array}{l}\text { Cryoneurolysis } \\
\text { of lumbar medial } \\
\text { branch nerves. }\end{array}$ & $\begin{array}{l}\text { Timing: } 6 \text { weeks, } \\
3,6 \text {, and } 12 \\
\text { months. } \\
\text { Outcome } \\
\text { measures: VAS to } \\
\text { ADLs measured } \\
\text { on four-step } \\
\text { simplified } \\
\text { McNab. }\end{array}$ & $\begin{array}{l}\text { Statistically significant } \\
\text { improvement in mean } \\
\text { low back pain of } \\
(\geq 50 \%) \text { at all points; } \\
\text { success or partial } \\
\text { success achieved in } \\
72 \% \text { of pts at } 6 \text { wks; } \\
70 \% \text { at } 3 \text { mos, and } 57 \% \\
\text { at } 12 \text { mos. }\end{array}$ & $\begin{array}{l}\text { Positive short- } \\
\text { term and long- } \\
\text { term relief }\end{array}$ \\
\hline $\begin{array}{l}\text { Staender et al (129) } \\
\text { Prospective evaluation } \\
\text { AHRQ score: } 5 / 8\end{array}$ & $\begin{array}{l}76 \text { patients with } \\
\text { chronic low } \\
\text { back pain with } \\
\text { a diagnosis of } \\
\text { lumbar facet } \\
\text { joint syndrome. } \\
\text { The diagnosis } \\
\text { was established } \\
\text { after } 3 \text { positive } \\
\text { CT-guided medial } \\
\text { branch nerve } \\
\text { blocks. }\end{array}$ & $\begin{array}{l}\text { Kryorhizotomy } \\
\text { of lumbar medial } \\
\text { branch nerves. }\end{array}$ & $\begin{array}{l}\text { Outcome } \\
\text { measures: VAS, } \\
\text { medication } \\
\text { usage, ability to } \\
\text { work, physical } \\
\text { conditions. } \\
\text { Timing: } 3 \text { days, } \\
3 \text { mos, and every } \\
3 \text { mos thereafter, } \\
\text { with a median } \\
\text { follow-up period } \\
\text { of } 22.5 \text { mos. }\end{array}$ & $\begin{array}{l}\text { Significant reduction } \\
\text { in the VAS at } 3 \\
\text { mos and } 6 \text { mos } \\
\text { were observed } \\
\text { postoperatively. In } 40 \% \\
\text { of patients, pain was } \\
\text { reduced for } 12 \text { mos } \\
\text { or longer. } 18 \text { patients } \\
\text { underwent a second, } \\
7 \text { patients underwent } \\
\text { a third, and } 1 \text { patient } \\
\text { underwent a fourth } \\
\text { procedure. }\end{array}$ & $\begin{array}{l}\text { Positive short- } \\
\text { term and long- } \\
\text { term relief }\end{array}$ \\
\hline
\end{tabular}


Pain Physician: January 2007:10:229-253

Table 6 Continued. Characteristics of results of studies of lumbar medial branch neurotomy

\begin{tabular}{|c|c|c|c|c|c|}
\hline Study/Methods & Participants & Intervention(s) & Outcome(s) & Result(s) & $\begin{array}{c}\text { Conclusion(s) } \\
\text { Short-term } \\
\text { relief }<3 \mathrm{mos} \\
\text { Long-term } \\
\text { relief } \geq 3 \mathrm{mos}\end{array}$ \\
\hline $\begin{array}{l}\text { Martinez-Suarez et al (131) } \\
\text { Retrospective evaluation } \\
\text { AHRQ score: } 4 / 8\end{array}$ & $\begin{array}{l}252 \text { pts with } \\
\text { diagnosis of } \\
\text { lumbar facet } \\
\text { joint pain were } \\
\text { included. }\end{array}$ & $\begin{array}{l}\text { Radiofrequency } \\
\text { medial branch } \\
\text { neurotomy. }\end{array}$ & Pain relief. & $\begin{array}{l}\text { Effectiveness in } 74.7 \% \\
\text { of cases. }\end{array}$ & $\begin{array}{l}\text { Positive short- } \\
\text { term and long- } \\
\text { term relief }\end{array}$ \\
\hline $\begin{array}{l}\text { North et al (149) } \\
\text { Retrospective } \\
\text { AHRQ score: } 6 / 8\end{array}$ & $\begin{array}{l}82 \text { pts with lumbar } \\
\text { pain of facet } \\
\text { origin diagnosed } \\
\text { by facet nerve } \\
\text { blocks. }\end{array}$ & $\begin{array}{l}\text { Radiofrequency } \\
\text { neurotomy of the } \\
\text { lumbar facets for } \\
42 \text { pts. }\end{array}$ & $\begin{array}{l}\text { Pain relief and } \\
\text { function. }\end{array}$ & $\begin{array}{l}45 \% \text { of pts with } \\
\text { radiofrequency } \\
\text { reported }>50 \% \text { pain } \\
\text { relief at } 2 \text { yrs. }\end{array}$ & $\begin{array}{l}\text { Negative short- } \\
\text { term and long- } \\
\text { term relief }\end{array}$ \\
\hline $\begin{array}{l}\text { Tzaan and Tasker (138) } \\
\text { Retrospective } \\
\text { AHRQ score: } 5 / 8\end{array}$ & $\begin{array}{l}90 \text { pts diagnosed } \\
\text { by local anesthetic } \\
\text { blocks. }\end{array}$ & $\begin{array}{l}\text { Radiofrequency } \\
\text { facet neurotomy } \\
\text { at involved levels. }\end{array}$ & Pain relief. & $\begin{array}{l}41 \% \text { of pts had }>50 \% \\
\text { relief at an average } \\
\text { follow-up of } 5.6 \\
\text { months. }\end{array}$ & $\begin{array}{l}\text { Negative short- } \\
\text { term and long- } \\
\text { term relief }\end{array}$ \\
\hline $\begin{array}{l}\text { Schaerer (139) } \\
\text { Retrospective } \\
\text { AHRQ score: } 5 / 8\end{array}$ & $\begin{array}{l}117 \text { pts } 71 \text { lumbar } \\
\text { procedures }\end{array}$ & $\begin{array}{l}\text { Radiofrequency } \\
\text { facet neurotomy } \\
\text { at involved levels. }\end{array}$ & $\begin{array}{l}\text { Pain, analgesics, } \\
\text { and mood. }\end{array}$ & $\begin{array}{l}35 \% \text { of } 71 \text { lumbar RF } \\
\text { had }>50 \% \text { pain relief, } \\
\text { with average follow-up } \\
\text { of } 13.7 \text { mos. }\end{array}$ & $\begin{array}{l}\text { Negative short- } \\
\text { term and long- } \\
\text { term relief }\end{array}$ \\
\hline $\begin{array}{l}\text { Schofferman and Kine } \\
(141) \\
\text { Retrospective } \\
\text { AHRQ Score: } 5 / 8\end{array}$ & $\begin{array}{l}20 \text { pts underwent } \\
\text { repeat } \\
\text { radiofrequency } \\
\text { neurotomy. } \\
\text { Medial branch } \\
\text { blocks used for } \\
\text { initial diagnosis. }\end{array}$ & $\begin{array}{l}\text { Radiofrequency } \\
\text { neurotomy } \\
\text { of the lumbar } \\
\text { facets; repeated } \\
\text { as indicated } \\
\text { clinically. }\end{array}$ & Pain relief. & $\begin{array}{l}\text { Mean duration of relief } \\
\text { of initial RF was } 10.5 \\
\text { mos. Second treatment } \\
\text { effective in } 85 \% \text {; mean } \\
\text { duration of relief of } \\
11.6 \text { mos. }\end{array}$ & $\begin{array}{l}\text { Positive short- } \\
\text { term and long- } \\
\text { term relief }\end{array}$ \\
\hline
\end{tabular}

cutaneous laser denervation in 15 patients in whom facet joint pain was confirmed by 2 diagnostic blocks. Of the 15 patients undergoing laser denervation, 8 experienced complete relief and 6 experienced more than $50 \%$ pain relief. They concluded that laser denervation was an effective treatment. This appears to be the first published report on laser denervation in a prospective study. Though selection criteria were appropriate, there were no outcome parameters and the results have not been substantiated in a randomized, double-blind trial.

Among the previously reviewed nonrandomized or observational studies, Dreyfuss et al (140) described lumbar facet joint radiofrequency neurotomy and found $60 \%$ improvement in $80 \%$ of patients at 1 year. McDonald et al (137) determined the long-term efficacy of percutaneous radiofrequency medial branch neurotomy for the treatment of chronic neck pain in 28 patients diagnosed as having cervical zygapophysial joint pain, on the basis of controlled diagnostic blocks. They reported a median duration of relief after the first procedure of 219 days when failures were included, and 422 days when only the successes were considered. In addition, radiofrequency neurotomy of the cervical zygapophysial joints significantly reduced headache severity in $80 \%$ of patients, both at shortterm and long-term follow-up.

Among the retrospective evaluations, MartinezSuarez et al (131) evaluated 252 patients with a diagnosis of lumbar facet joint pain with radiofrequency neurotomy of medial branches. They reported effectiveness in $74.7 \%$ of cases. Tzaan and Tasker (138) evaluated 118 consecutive percutaneous radiofrequency facet rhizotomies performed on 90 patients for cervical, thoracic, and lumbar pain. They reported that with the first procedure, greater than $50 \%$ subjective reduction of pain was present in $41 \%$ of patients; this was considered a negative outcome. They included cervical, thoracic, and lumbosacral facets and noted no significant difference between unilateral or 
Table 7. Characteristics of results of studies of thoracic medial branch neurotomy.

\begin{tabular}{|c|c|c|c|c|c|}
\hline Study/Methods & Participants & Intervention(s) & Outcome(s) & Result(s) & $\begin{array}{c}\text { Conclusion(s) } \\
\text { Short-term } \\
\text { relief }<3 \text { mos } \\
\text { Long-term } \\
\text { relief } \geq 3 \text { mos }\end{array}$ \\
\hline $\begin{array}{l}\text { Tzaan and Tasker (138) } \\
\text { Retrospective } \\
\text { AHRQ score: } 5 / 8\end{array}$ & $\begin{array}{l}90 \text { pts diagnosed } \\
\text { by local anesthetic } \\
\text { blocks. }\end{array}$ & $\begin{array}{l}\text { Radiofrequency } \\
\text { facet neurotomy } \\
\text { at involved } \\
\text { levels, including } \\
\text { thoracic. }\end{array}$ & Pain relief. & $\begin{array}{l}41 \% \text { of pts had }>50 \% \text { relief at } \\
\text { an average follow-up of } 5.6 \\
\text { months. }\end{array}$ & $\begin{array}{l}\text { Negative short- } \\
\text { term and long- } \\
\text { term relief }\end{array}$ \\
\hline $\begin{array}{l}\text { Stolker et al (150) } \\
\text { Retrospective } \\
\text { AHRQ score: } 5 / 8\end{array}$ & $\begin{array}{l}40 \text { pts with } \\
\text { chronic thoracic } \\
\text { facet pain } \\
\text { diagnosed with } \\
\text { medial branch } \\
\text { block. }\end{array}$ & $\begin{array}{l}\text { Thoracic } \\
\text { radiofrequency } \\
\text { facet neurotomy. }\end{array}$ & Pain relief. & $\begin{array}{l}\text { At an average follow-up of } 31 \\
\text { months, } 83 \% \text { had }>50 \% \text { relief. }\end{array}$ & $\begin{array}{l}\text { Positive short- } \\
\text { term and long- } \\
\text { term relief }\end{array}$ \\
\hline
\end{tabular}

bilateral involvement. On the other hand, the retrospective study by Stolker et al (150) was positive for thoracic facets.

North et al (149) evaluated radiofrequency lumbar facet denervation with long-term outcome assessment by a disinterested third party interview. Forty-five percent of patients undergoing denervation reported at least $50 \%$ relief of pain at long-term follow-up. Schaerer (139) evaluated the value of radiofrequency facet rhizotomy in the treatment of patients with chronic neck and low back pain problems in 90 consecutive patients, undergoing 117 procedures. They reported that overall results were good to excellent in $50 \%$ of the patients in cervical spine and $35 \%$ in lumbar spine with an average follow-up time of 13.7 months. Schofferman and Kine (141) demonstrated that radiofrequency neurolysis can be repeated when pain returns, re-establishing long-term pain relief in $85 \%$ of patients.

Criticisms faced by these trials include the overall relatively small number of patients. The total number of patients combined for cervical and lumbar regions in the randomized trials was 27 in the treatment groups, compared to 28 in the control groups. Consequently, the number of patients undergoing a cervical or lumbar intervention was small. An additional criticism for van Kleef's et al's (135) study is that they included patients after a single diagnostic block, which may increase the false-positive rate. Prospective studies also had a small total number of patients.

\section{Cost Effectiveness}

No studies were performed evaluating cost effectiveness of medial branch neurotomy.

\section{Level of Evidence}

No new randomized trial was included in the present analysis, because of technical difficulties with the one identified randomized study. However, several new observational studies were included in the present analysis, including a report on cryoneurolysis of lumbar medial branches. The evidence for neurotomy (radiofrequency and cryoneurolysis) is moderate or Level III for long-term relief of cervical and lumbar facet joint pain. This is a change from our previous systematic review (1), where evidence was moderate to strong for shortand long-term relief. However, we would like to emphasize that cervical medial branch radiofrequency performed as advocated by Bogduk (151), and as reported in a randomized study by Lord et al (134) and prospective evaluations by McDonald et al (137) and Barnsley (126), involves producing multiple lesions at each medial branch location in the cervical spine, with significantly longer duration of operative time. This technique is not routinely practiced in the United States. But, with this technique, the evidence for cervical medial branch radiofrequency neurotomy is considered strong for short-term and long-term relief. For all other techniques, the evidence is strong for short-term and moderate for long-term relief in managing lumbar and cervical facet joint pain with radiofrequency thermoneurolysis. The evidence for thoracic medial branch radiofrequency is indeterminate.

The evidence is indeterminate for pulsed radiofrequency and laser medial branch neurotomy. 


\section{Safety and Complications}

Our search strategy (complications/facet injections/denervation/radiofrequency) yielded 6 new articles. Several additional references were identified during searches for facet interventions. Septic joints and transient tetraplegia have been reported after intraarticular facet injections $(152,153)$. Following radiofrequency denervation, post-procedural MRI findings can demonstrate contrast enhancement typical of that seen with a paraspinal abscess, even without apparent infection (154). The authors suggested that pain after radiofrequency may be due to a noninfectious postinflammatory process and may not warrant antibiotic treatment unless indicated on clinical grounds (154).

The risks associated with needle injections may be higher in the cervical spine, particularly with intraarticular facet joint injections and perhaps medial branch blocks. Although quantitative data are lacking, a recent anatomic study by Huntoon (155) demonstrated that the deep cervical artery lies adjacent to the upper cervical facets and may anastomose with the circulation of the cervical spinal cord in about $10 \%$ of individuals. Cervical arteries may be vulnerable during soft tissue injections adjacent to the cervical spine. The risk of such a complication is supported by a recent case report by Heckmann et al (153), who noted transient tetraplegia after a cervical facet joint injection for chronic neck pain done without imaging. Immediately following a presumed facet injection at the $\mathrm{C} 6$ level guided by anatomical landmarks, the patient developed tetraplegia that lasted for about 30 minutes. Proprioception remained intact throughout. The patient made a rapid and complete recovery. The authors surmised that an inadvertent injection of local anesthetic into a radicular artery feeding the anterior spinal artery temporally compromised the cervical spinal cord. Although the precise mechanism is speculative in this case given the lack of fluoroscopic imaging, cervical facet injections may be associated with major complications.

The most common and worrisome complications of facet joint interventions were related to needle placement and drug administration. Potential complications include dural puncture, spinal cord trauma, infection, intraarterial or intravenous injection, spinal anesthesia, chemical meningitis, neural trauma, pneumothorax, radiation exposure, facet capsule rupture, hematoma formation, and steroid side effects (156-172).

Potential side effects with radiofrequency denervation include painful cutaneous dysesthesias, in- creased pain due to neuritis or neurogenic inflammation, anesthesia dolorosa, cutaneous hyperesthesia, pneumothorax, and deafferentation pain. Unintentional damage to a spinal nerve during medial branch radiofrequency, causing a motor deficit, is also a possible complication of a neurolytic procedure.

A retrospective chart review of patients undergoing radiofrequency neurotomy for lumbar facet joint pain over a 5-year period provided complication rates associated with the procedure (171). During 116 separate denervation procedures, 6 minor complications were noted, including 3 cases of localized pain lasting $>2$ weeks and 3 cases of neuritic pain lasting $<2$ weeks. In that study, there were no cases of infection or new sensory or motor deficits. The overall rate of complications, which were minor, was $1 \%$.

\section{Discussion}

This systematic review provides an update of the evidence for the effectiveness of facet interventions for the management of chronic facet joint spinal pain. Only 2 new studies were included in the evidence synthesis for intraarticular facet joint injections. Although the study by Gorbach et al (101) was technically satisfactory based on AHRQ criteria, the diagnosis of facet pain was made on clinical grounds and not on the basis of diagnostic blocks. Although the results were positive for the value of intraarticular facet injections for short-term relief, the lack of diagnostic blocks as a requirement for patient inclusion was deemed to be a significant criticism of the study. Therefore, the study was not included in the current evidence synthesis.

The study by Fuchs et al (100) evaluated the efficacy and safety of intraarticular sodium hyaluronate (SH) as compared with intraarticular triamcinolone acetonide in the treatment of chronic nonradicular lumbar pain. They included 60 patients in a randomized, controlled, blind-observer clinical study and randomly assigned patients to receive $10 \mathrm{mg}$ of SH or 10 mg TA per facet joint. The facet joints on both sides at levels L3/4, L4/5, and L5/S1 were treated once per week under computed tomographic guidance, for a total of 6 injections. The study demonstrated significant pain relief, improved function, and quality of life with both treatments; intraarticular hyaluronate was equivalent to intraarticular steroid, for a period of up to 6 months. The drawbacks of this study include lack of diagnosis of facet joint pain by controlled local anesthetic blocks, which may have increased the probability of inclusion of patients without facet joint pain. 
A second report, a prospective observational study by Kim et al (103) was included in the evidence synthesis. Patients with cervical facet joint pain were treated with therapeutic facet joint injections with lidocaine and triamcinolone under fluoroscopy. Patients were classified into a herniated nucleus pulposus group, myofascial pain syndrome group, and whiplash-associated disorders group. The symptomfree duration after blockade lasted longer in the herniated nucleus pulposus group (11.3 \pm 1.7 months). The authors suggested that perpetuating factors associated with myofascial and whiplash pain may not respond as well as pain associated with disc degeneration. The results do suggest that intraarticular cervical facet injections may be helpful for facet pain associated with disc herniation.

In the previous systematic review regarding intraarticular facet joint injections, only the randomized trial of lumbar spine pain by Carette et al (104) was considered positive, with a $42 \%$ success rate. In contrast, a randomized trial of cervical spine pain following whiplash injury by Barnsley et al (108) was negative. Among the nonrandomized trials, positive results were noted for short-term relief in 5 of the 6 studies. However, long-term relief was noted in only 4 of the 6 studies.

The evidence for the effectiveness of intraarticular facet injections is moderate for short- and long-term relief of lumbar facet pain. The only randomized trial of cervical spine facet joint injections was negative, although there are 2 positive observational studies available $(103,115)$. Based on the previous analysis and with 2 new studies, the evidence for the effectiveness of cervical intraarticular facet joint injections is limited for short- and long-term relief.

Regarding medial branch blocks for management of facet joint pain, 3 new studies evaluated the effectiveness of medial branch blocks for chronic facet pain. Manchikanti et al (116) evaluated pain relief with cervical medial branch blocks in a randomized, double-blind, controlled trial using four types of injections. Significant pain relief (> 50\%) and improvement in functional status were observed at 3,6, and 12 months. The average number of treatments for 1 year was approximately 3 to 4 injections, with no difference between treatment groups. Duration of relief was approximately 14 weeks per treatment. Although there was no placebo control group in the study, the results demonstrated that medial branch nerve blocks, with or without steroid, could provide predictable pain relief. Inclusion of betamethasone in the injectate did not appear to provide additional benefit. This study provides further evidence that cervical facet joint medial branch blocks may be considered therapeutic in nature and with repeated injections may be a means of providing durable relief of facet pain without neurolysis.

In the second new study, Manchikanti et al (117) evaluated 60 patients in a randomized, double-blind, controlled trial. Patients with lumbar facet pain diagnosed with comparative local anesthetic medial branch blocks were randomized to receive therapeutic local anesthetic medial branch blocks of lumbar facets, with or without steroid (betamethasone). Statistically significant pain relief and functional status improvement were observed at 3 months, 6 months, and 12 months, compared to baseline measurements. The average number of treatments per patient for 1 year was approximately 3.5 injections, with no significant differences noted between treatment groups. Duration of average pain relief with each procedure session was about 3.5 months. This small, randomized study provides additional evidence that medial branch blocks may be considered therapeutic for patients with chronic low back pain of facet origin.

The third new study (118) examined the therapeutic benefit of thoracic medial branch blocks in a prospective outcome study. In 55 consecutive patients with thoracic facet pain confirmed by comparative diagnostic facet nerve blocks, more than two-thirds of patients obtained significant pain relief (> 50\%) with bupivacaine and methylprednisolone at 3, 6, 12, 24, and 36 months, compared to baseline measurements. Patients received approximately 3 to 4 blocks per year with an average duration of relief per treatment of about 4 months. As with the cervical facet nerve block report, this study provides evidence that repeated medial branch blocks may provide durable relief of spinal pain of thoracic facet joint origin.

Based on the 3 new studies and the previously reviewed reports for medial branch facet nerve blocks, evidence for relief of chronic cervical, thoracic, and lumbar facet pain is considered moderate for shortand long-term pain relief. Based on best evidence, long-term relief of facet joint pain with medial branch blocks may require local anesthetic injections at intervals of approximately 3 to 4 months, with or without steroid.

Regarding radiofrequency neurotomy for lumbar facet pain, a randomized trial by van Wijk and colleagues (123) was identified for the current sys- 
tematic review. The study was of high quality, based on AHRQ and Cochrane criteria; however the study was subject to criticism on technical grounds. Bogduk (124) noted that the radiofrequency needle position was improper, with needle active tips located too far lateral to the medial branch nerves to ensure denervation. This could have increased the likelihood of treatment failure. It should also be noted that single diagnostic blocks were used with a criterion of $50 \%$ pain relief, rather than comparative controlled diagnostic blocks with $75 \%$ or $80 \%$ pain relief, which may have increased the false-positive rate. This may have reduced the likelihood of noting a statistical effect with active treatment. Based on these limitations, the study was excluded from further analysis for the current review.

A prospective outcome study of cervical facet radiofrequency by Barnsley (126); a prospective study by Shin et al (127) of pain relief with lumbar facet radiofrequency; a retrospective study of lumbar pain by Martinez-Suarez et al (131); and 2 studies by Birkenmaier et al (128) and Staender et al (129) of cryodenervation of lumbar facets met inclusion criteria and were included in the present analysis. These studies were positive for short- and long-term relief of facet pain.

From our previous systematic review, 2 randomized trials, 1 of the cervical spine (134) and 1 involving the lumbar spine (135), met inclusion criteria and are included in the present analysis. Both of these studies, which involved small numbers of patients, were positive for short- and long-term pain relief.

The 4 observational studies of cervical medial branch neurotomy (136-139) from the previous systematic review are included in the current analysis, 2 of which were positive for short- and long-term pain relief. The 6 observational studies of lumbar medial branch neurotomy $(138,139,140-143)$ from the previous review met criteria for inclusion in the current analysis. Of these, 3 studies were positive for shortand long-term pain relief. Two observational studies of thoracic medial branch neurotomy $(138,150)$ met inclusion criteria in the previous systematic review and are included in the current analysis. Of these 2 studies, 1 was positive for short- and long-term pain relief (150).

Regarding radiofrequency neurolysis for chronic facet pain, no new randomized trial was included in evidence synthesis, because of technical limitations of the 1 new randomized study (123). However, several new observational studies met inclusion criteria and were incorporated into evidence synthesis, including a report on cryoneurolysis of lumbar medial branches.

Based on current information, the evidence for neurotomy (radiofrequency and cryoneurolysis) is moderate for short- and long-term relief of lumbar and cervical facet joint pain and indeterminate for thoracic facet pain. If the denervation protocol used by Lord et al (134), McDonald et al (137) and Barnsley (126) is followed, which is extensive and involves producing multiple lesions of each facet nerve, the evidence is strong for short- and long-term relief of pain with cervical medial branch neurotomy.

\section{Conclusion}

Based on a systematic review of the included studies described herein, for cervical intraarticular facet joint injections, the evidence is limited for short- and long-term pain relief. For lumbar intraarticular facet joint injections, there is moderate evidence for shortand long-term pain relief.

For cervical, thoracic and lumbar medial branch nerve blocks, the evidence is moderate for short- and long-term pain relief.

The evidence for neurotomy (radiofrequency and cryodenervation) of cervical and lumbar medial branch nerves is moderate for short- and long-term pain relief. The evidence is strong for cervical medial branch neurotomy if the procedure is performed with multiple lesions, as described by Lord et al (134), McDonald et al (137), and Barnsley (126). The evidence for thoracic medial branch neurotomy is indeterminate. 


\section{References}

1. Boswell MV, Colson JD, Spillane WF. Therapeutic facet joint interventions in chronic spinal pain: a systematic review of effectiveness and complications. Pain Physician 2005; 8:101-114.

2. Sehgal N, Shah RV, McKenzie-Brown $A$, Everett CR. Diagnostic utility of facet (zygapophysial) joint injections in chronic spinal pain: a systematic review of evidence. Pain Physician 2005; 8:211-224.

3. Fukui S, Ohseto K, Shiotani M, Ohno K, Karasawa H, Naganuma Y, Yuda Y. Referred pain distribution of the cervical zygapophyseal joints and cervical dorsal rami. Pain 1996; 68:79-83.

4. Dwyer A, Aprill C, Bogduk N. Cervical zygapophyseal joint pain patterns: a study in normal volunteers. Spine 1990; 15:453-457.

5. Aprill C, Dwyer A, Bogduk N. The prevalence of cervical zygapophyseal joint pain patterns II: a clinical evaluation. Spine 1990; 15:458-461.

6. Pawl RP. Headache, cervical spondylosis, and anterior cervical fusion. Surg Ann 1977; 9:391-498.

7. Windsor RE, Nagula D, Storm S. Electrical stimulation induced cervical medial branch referral patterns. Pain Physician 2003; 6:411-418.

8. Dreyfuss P, Tibiletti C, Dreyer SJ. Thoracic zygapophyseal joint pain patterns: a study in normal volunteers. Spine 1994; 19:807-811.

9. Fukui S, Ohseto K, Shiotani M. Patterns of pain induced by distending the thoracic zygapophyseal joints. Reg Anesth 1997; 22:332-336.

10. Mooney V, Robertson J. The facet syn drome. Clin Orthop 1976; 115:149-156.

11. McCall IW, Park WM, O'Brien JP. Induced pain referral from posterior elements in normal subjects. Spine 1979; 4:441-446.

12. Marks R. Distribution of pain provoked from lumbar facet joints and related structures during diagnostic spinal infiltration. Pain 1989; 39:37-40.

13. Fukui S, Ohseto K, Shiotani M, Ohno K, Karasawa H, Naganuma Y. Distribution of referral pain from the lumbar zyg. apophyseal joints and dorsal rami. Clin J Pain 1997; 13:303-307.

14. Hirsch C, Ingelmark BE, Miller M. The anatomical basis for low back pain. Acta Orthop Orthop Scand 1963; 33:1-17.

15. Windsor RE, King FJ, Roman SJ, Tata N, Cone-Sullivan LA, Thampi S, Acebey M, Gilhool JJ, Rao R, Sugar R. Electri- cal stimulation induced lumbar medial branch referral patterns. Pain Physician 2002; 5:347-353.

16. Bogduk N. The clinical anatomy of the cervical dorsal rami. Spine 1982; 7:319330.

17. Cavanaugh JM, Ozaktay AC, Yamashita T, Avramov A, Getchell TV, King Al. Mechanisms of low back pain: a neurophysiologic and neuroanatomic study. Clin Orthop 1997; 335:166-180.

18. Bogduk N, Wilson AS, Tynan W. The human lumbar dorsal rami. J Anat 1982; 134:383-397.

19. Ohtori S, Takahashi K, Chiba T, Yamagata M, Sameda H, Moriya H. Sensory innervation of the cervical facet joints in rats. Spine 2001; 26:147-150.

20. Chua WH, Bogduk N. The surgical anatomy of thoracic facet denervation. Acta Neurochir 1995; 136:140-144.

21. Barnsley L, Bogduk N. Medial branch blocks are specific for the diagnosis of cervical zygapophyseal joint pain. Reg Anesth 1993; 18:343-350.

22. Zhang J, Tsuzuki N, Hirabayashi S, Saiki K, Fujita K. Surgical anatomy of the nerves and muscles in the posterior cervical spine. Spine 2003; 1379-1384.

23. Dreyfuss P, Schwarzer AC, Lau P, Bogduk N. Specificity of lumbar medial branch and $L_{5}$ dorsal ramus blocks. Spine 1997; 22:895-902.

24. Kaplan M, Dreyfuss P, Halbrook B, Bogduk N. The ability of lumbar medial branch blocks to anesthetize the zygapophysial joint. Spine 1998; 23:18471852.

25. Özaktay AC, Yamashita T, Cavanaugh JM, King Al. Fine nerve fibers and endings in the fibrous capsule of the lum. bar facet joint. Trans Orthop Res Soc 1991; 16:353.

26. Ishikawa T, Miyagi M, Ohtori S, Aoki Y, Ozawa T, Doya H, Saito T, Moriya H, Takahashi K. Characteristics of sensory DRG neurons innervating the lumbar facet joints in rats. Eur Spine J 2005; 14:559-564.

27. Suseki K, Takahashi Y, Takahashi K, Chiba T, Tanaka K, Morinaga T, Nakamura S, Moriya $\mathrm{H}$. Innervation of the lumbar facet joints. Origins and functions. Spine 1997; 22:477-485.

28. Sameda H, Takahashi Y, Takahashi K, Chiba T, Ohtori S, Moriya H. Primary sensory neurons with dichotomizing axons projecting to the facet joint and the sciatic nerve in rats. Spine 2001; 26:1105-1109.
29. Yamada H, Honda T, Kikuchi S, Sugiura $Y$. Direct innervation of sensory fibers from the dorsal root ganglion of the cervical dura mater of rats. Spine 1998; 23:1524-1529.

30. Johnson GM. The sensory and sympathetic nerve supply within the cervical spine: review of recent observations. Man Ther 2004; 9:71-76.

31. Yoganandan N, Knowles SA, Maiman DJ, Pintar FA. Anatomic study of the morphology of human cervical facet joint. Spine 2003; 28:2317-2323.

32. Konnai Y, Honda T, Sekiguchi Y, Kikuchi $S$, Sugiura Y. Sensory innervation of the lumbar dura mater passing through the sympathetic trunk in rats. Spine 2000; 25:776-782.

33. Masini M, Paiva WS, Araujo AS Jr. Anatomical description of the facet joint innervation and its implication in the treatment of recurrent back pain. J Neurosurg Sci 2005; 49:143-146.

34. Ahmed M, Bjurholm A, Kreicbergs A, Schultzberg M. Sensory and autonomic innervation of the facet joint in the rat lumbar spine. Spine 1993; 18:21212126.

35. Cavanaugh JM, Lu Y, Chen C, Kallakuri $S$. Pain generation in lumbar and cervical facet joints. I Bone Joint Surg Am 2006; 88 Suppl 2:63-67.

36. Inami S, Shiga T, Tsujino A, Yabuki T, Okado N, Ochiai N. Immunohistochemical demonstration of nerve fibers in the synovial fold of the human cervical facet joint. J Orthop Res 2001; 19:593-596.

37. Kallakuri S, Singh A, Chen C, Cavanaugh JM. Demonstration of substance P, calcitonin gene-related peptide, and protein gene product 9.5 containing nerve fibers in human cervical facet joint capsules. Spine 2004; 29:1182-1186.

38. Chen C, Lu Y, Kallakuri S, Patwardhan A, Cavanaugh JM. Distribution of A-delta and $\mathrm{C}$-fiber receptors in the cervical facet joint capsule and their response to stretch. J Bone Joint Surg Am 2006; 88:1807-1816.

39. Ohtori S, Takahashi K, Moriya H. Calcitonin gene-related peptide immunoreactive DRG neurons innervating the cervical facet joints show phenotypic switch in cervical facet injury in rats. Eur Spine J 2003; 12:211-215.

40. Miyagi M, Ohtori S, Ishikawa T, Aoki Y, Ozawa T, Doya H, Saito T, Moriya H, Takahashi K. Up-regulation of TNFalpha in DRG satellite cells following lumbar facet joint injury in rats. Eur Spine J 2006; 15:953-958. 
41. Ohtori S, Takahashi K, Chiba T, Yamagata M, Sameda H, Moriya H. Substance $P$ and calcitonin gene-related peptide immunoreactive sensory DRG neurons innervating the lumbar facet joints in rats. Auton Neurosci 2000; 86:13-17.

42. Suseki K, Takahashi Y, Takahashi K, Chiba T, Tanaka K, Moriya H. CGRPimmunoreactive nerve fibers projecting to lumbar facet joints through the paravertebral sympathetic trunk in rats. Neurosci Lett 1996; 221:41-44.

43. Ohtori S, Moriya H, Takahashi K. Calcitonin gene-related peptide immunoreactive sensory DRG neurons innervating the cervical facet joints in rats. J Orthop Sci 2002; 7:258-261.

44. Ohtori S, Takahashi K, Chiba T, Yamagata M, Sameda H, Moriya H. Brain-derived neurotrophic factor and vanilloid receptor subtype 1 immunoreactive sensory DRG neurons innervating the lumbar facet joints in rats. Auton Neurosci 2001; 94:132-135.

45. McLain RF, Pickar JG. Mechanoreceptor endings in human thoracic and lumbar facet joints. Spine 1998; 23:168-173.

46. McLain RF. Mechanoreceptors ending in human cervical facets joints. Spine 1994; 5:495-501.

47. Yamashita T, Cavanaugh JM, el-Bohy AA, Getchell TV, King Al. Mechanosensitive afferent units in the lumbar facet joint. J Bone Joint Surg (Am) 1990; 72:865-870.

48. Yamashita T, Cavanaugh JM, Ozaktay AC, Avramov Al, Getchell TV, King Al. Effect of substance P on mechanosensitive units of tissues around and in the lumbar facet joint. J Orthop Res 1993; 11:205-214.

49. Beaman DN, Graziano GP, Glover RA, Wojtys EM, Chang V. Substance P innervation of lumbar spine facet joints. Spine 1993; 18:1044-1049.

50. Ohtori S, Takahashi K, Chiba T, Yamagata M, Sameda H, Moriya H. Phenotypic inflammation switch in rats shown by calcitonin gene-related peptide immunoreactive dorsal root ganglion neurons innervating the lumbar facet joints. Spine 2001; 26:1009-1013.

51. Chen C, Lu Y, Cavanaugh JM, Kallakuri S, Patwardhan A. Recording of neural activity from goat cervical facet joint capsule using custom-designed miniature electrodes. Spine 2005; 30:13671372.

52. Lu Y, Chen C, Kallakuri S, Patwardhan A, Cavanaugh JM. Development of an in vivo method to investigate biomechanical and neurophysiological prop- erties of spine facet joint capsules. Eur Spine J 2005; 14:565-572.

53. Lu Y, Chen C, Kallakuri S, Patwardhan A, Cavanaugh JM. Neurophysiological and biomechanical characterization of goat cervical facet joint capsules. J Orthop Res 2005; 23:779-787.

54. Mayer TG, Gatchel RJ, Keeley J, McGeary D, Dersh J, Anagnostis C. A randomized clinical trial of treatment for lumbar segmental rigidity. Spine 2004; 29:2199-2205.

55. El-Bohy AA, Goldberg SJ, King Al. Measurement of facet capsular stretch. In: Proceedings of the American Society of Mechanical Engineers, Bioengineering Symposium; New York, NY, Jun 14-17, 1987, pp 161-164.

56. Yang KH, King Al. Mechanism of facet load transmission as a hypothesis for low-back pain. Spine 1984; 9:557-565.

57. Borchgrevink GE, Smevik O, Nordby A, Rinck PA, Stiles TC, Lereim I. MR imag ing and radiography of patients with cervical hypertension-flexion injuries after car accidents. Acta Radiol 1995; 36:425-428.

58. Ronnen HR, de Korte PJ, Brink PR, van der Bijl HJ, Tonino AJ, Franke CL. Acute whiplash injury: is there a role for MR imaging? - a prospective study of 100 patients. Radiology 1996; 201:93-96.

59. Pennie B, Agambar L. Patterns of injury and recovery in whiplash. Injury 1991; 22:57-59.

6o. Siegmund GP, Myers BS, Davis MB, Bohnet HF, Winkelstein BA. Human cervical motion segment flexibility and facet capsular ligament strain under combined posterior shear, extension and axial compression. Stapp Car Crash J 2000; 44:159-170.

61. Winkelstein BA, Nightingale RW, Richardson WJ, Myers BS. Cervical facet joint mechanics: its application to whiplash injury. Stapp Car Crash J 1999; 43:243-252.

62. Onan OA, Heggeness MH, Hipp JA. A motion analysis of the cervical facet joint. Spine 1998; 23:430-439.

63. Stemper BD, Yoganandan N, Pintar FA. Gender- and region-dependent local facet joint kinematics in rear impact: implications in whiplash injury. Spine 2004; 29:1764-1771.

64. Keeley J, Mayer T, Cox R, Gatchel RJ, Smith J, Mooney V. Quantification of lumbar function: part 5. Reliability of range of motion measures in the sagittal plane and an in vivo torso rotation measurement technique. Spine 1986; 11:31-35.
65. Mayer T, Robinson R, Pegues P, Kohles $S$, Gatchel RJ. Lumbar segmental rigidity: can its identification with facet injections and stretching exercises be useful? Arch Phys Med Rehabil 2000; 81:1143-1150.

66. Merskey H, Bogduk N. Classification of chronic pain. In: Descriptions of Chronic Pain Syndromes and Definition of Pain Terms, 2nd ed. IASP Press, Seattle, WA, 1994, pp 180-181.

67. Bogduk N. Low back pain. In: Clinical Anatomy of the Lumbar Spine and Sacrum, 4th ed. Churchill Livingstone, New York, NY, 2005, pp 183-216.

68. Bogduk N. International Spinal Injection Society guidelines for the performance of spinal injection procedures. Part 1: Zygapophyseal joint blocks. Clin J Pain 1997; 13:285-302.

69. Boswell MV, Singh V, Staats PS, Hirsch JA. Accuracy of precision diagnostic blocks in the diagnosis of chronic spinal pain of facet or zygapophysial joint origin. Pain Physician 2003; 6:449-456.

70. Schwarzer AC, Aprill CN, Derby R, Fortin J, Kine G, Bogduk N. Clinical features of patients with pain stemming from the lumbar zygapophysial joints. Is the lumbar facet syndrome a clinical entity? Spine 1994; 19:1132-1137.

71. Manchikanti L, Pampati V, Fellows B, Pakanati RR. Prevalence of lumbar facet joint pain in chronic low back pain. Pain Physician 1999; 2:59-64.

72. Manchikanti L, Pampati V, Fellows B, Baha A. The inability of the clinical picture to characterize pain from facet joints. Pain Physician 2000; 3:158-166.

73. Manchikanti L, Pampati V, Fellows B, Bakhit $C$. The diagnostic validity and therapeutic value of medial branch blocks with or without adjuvants. Curr Rev Pain 2000; 4:337-344.

74. Schwarzer AC, Wang SC, Bogduk N, MCNaught PJ, Laurent R. Prevalence and clinical features of lumbar zygapophysial joint pain: a study in an Australian population with chronic low back pain. Ann Rheum Dis 1995; 54:100-106.

75. Manchikanti L, Singh V, Pampati V, Damron K, Barnhill R, Beyer C, Cash K. Evaluation of the relative contributions of various structures in chronic low back pain. Pain Physician 2001; 4:308-316.

76. Manchikanti L, Hirsch JA, Pampati V. Chronic low back pain of facet (zygapophysial) joint origin: is there a difference based on involvement of single or multiple spinal regions? Pain Physician 2003; 6:399-405. 
77. Manchikanti L, Singh V, Pampati V, Damron K, Beyer C, Barnhill R. Is there correlation of facet joint pain in lumbar and cervical spine? An evaluation of prevalence in combined chronic low back and neck pain. Pain Physician 2002; 5:365-371.

78. Manchikanti L, Boswell MV, Singh V, Pampati V, Damron KS, Beyer CD. Prevalence of facet joint pain in chronic spinal pain of cervical, thoracic, and lumbar regions. BMC Musculoskelet Disord 2004; 5:15.

79. Manchukonda R, Manchikanti KN, Cash KA, Pampati V, Manchikanti L. Facet joint pain in chronic spinal pain: an evaluation of prevalence and false positive rate of diagnostic blocks. 2007; in press.

8o. Manchikanti L, Manchukonda R, Pampati V, Damron KS, McManus CD. Prevalence of facet joint pain in chronic low back pain in post surgical patients by controlled comparative local anesthetic blocks. Arch Phys Med Rehabil 2007; in press.

81. Bogduk N, Lord S. Cervical zygapophysial joint pain. Neurosurgery 1998; 8:107-117.

82. Barnsley L, Lord SM, Wallis BJ, Bogduk $\mathrm{N}$. The prevalence of chronic cervical zygapophyseal joint pain after whiplash. Spine 1995; 20:20-26.

83. Lord SM, Barnsley L, Wallis BJ, Bogduk N. Chronic cervical zygapophysial joint pain with whiplash: A placebo-controlled prevalence study. Spine 1996; 21:1737-1745.

84. Manchikanti L, Singh V, Rivera J, Pampati V. Prevalence of cervical facet joint pain in chronic neck pain. Pain Physician 2002; 5:243-249.

85. Speldewinde GC, Bashford GM, Davidson IR. Diagnostic cervical zygapophyseal joint blocks for chronic cervical pain. Med J Aust 2001; 174:174-176.

86. Barnsley L, Lord S, Wallis B, Bogduk N. False-positive rates of cervical zygapophysial joint blocks. Clin J Pain 1993; 9:124-130.

87. Manchikanti L, Singh V, Pampati V, Beyer C, Damron K. Evaluation of the prevalence of facet joint pain in chronic thoracic pain. Pain Physician 2002; 5:354-359.

88. Geurts JW, van Wijk RM, Stolker RJ, Groen GJ. Efficacy of radiofrequency procedures for the treatment of spinal pain: a systematic review of randomized clinical trials. Reg Anesth Pain Med 2001; 26:394-400.
89. Niemisto L, Kalso E, Malmivaara A, Seitsalo S, Hurri H, Cochrane Collaboration Back Review Group. Radiofrequency denervation for neck and back pain: a systematic review within the framework of the Cochrane collaboration back review group. Spine 2003 , 28:1877-1888.

90. Airaksinen O, Brox JI, Cedraschi C, Hildebrandt J, Klaber-Moffett J, Kovacs F, Mannion AF, Reis S, Staal JB, Ursin H, Zanoli G. Chapter 4: European guidelines for the management of chronic nonspecific low back pain. Eur Spine J 2006; 15:S192-S300.

91. Manchikanti L, Singh V, Vilims BD, Hansen HC, Schultz DM, Kloth DS. Medial branch neurotomy in management of chronic spinal pain: Systematic review of the evidence. Pain Physician 2002; 5:405-418.

92. Slipman CW, Bhat AL, Gilchrist RV, Issac $Z$, Chou L, Lenrow DA. A critical review of the evidence for the use of zygapophysial injections and radiofrequency denervation in the treatment of low back pain. Spine J 2003; 3:310-316.

93. Nelemans PJ, deBie RA, deVet HC, Sturmans $F$. Injection therapy for subacute and chronic benign low back pain. Spine 2001; 26:501-515.

94. Bogduk N. A narrative review of intraarticular corticosteroid injections for low back pain. Pain Med 2005; 6:287-296.

95. Manchikanti L, Abdi S, Lucas LF. Evidence synthesis and development of guidelines in interventional pain management. Pain Physician 2005; 8:73-86.

96. Manchikanti L, Heavner J, Racz GB, Mekhail NA, Schultz DM, Hansen HC, Singh V. Methods for evidence synthesis in interventional pain management. Pain Physician 2003; 6:89-111.

97. West S, King V, Carey TS, Lohr KN, McKoy N, Sutton SF, Lux L. Systems to rate the strength of scientific evidence. Evidence Report/Technology Assessment No. 47 University of North Carolina: Agency for Healthcare Research and Quality. AHRQ Publication No. 02-E016; April 2002.

98. van Tulder M, Assendelft W, Koes B, Bouter LM. Method guidelines for systematic reviews in the Cochrane Collaboration Back Review Group for Spinal Disorders. Spine 1997; 22:23232330.

99. Shekelle PG, Andersson G, Bombardier C, Cherkin D, Deyo R, Keller R, Lee C, Liang M, Lipscomb B, Spratt K. A brief introduction to the critical reading of the clinical literature. Spine 1994; 19(suppl):2028S-2031S.

100. Fuchs S, Erbe T, Fischer HL, Tibesku $\mathrm{CO}$. Intraarticular hyaluronic acid versus glucocorticoid injections for nonradicular pain in the lumbar spine. J Vasc Interv Radiol 2005; 16:1493-1498.

101. Gorbach C, Schmid MR, Elfering A, Hodler J, Boos N. Therapeutic efficacy of facet joint blocks. AJR Am J Roentgenol 2006; 186:1228-1233.

102. Shih C, Lin G-Y, Yueh K-C, Lin J-J. Lumbar zygapophyseal joint injections in patients with chronic lower back pain. J Chin Med Assoc 2005; 68:59-64.

103. Kim KH, Choi SH, Kim TK, Shin SW, Kim $\mathrm{CH}$, Kim Jl. Cervical facet joint injections in the neck and shoulder pain. J Korean Med Sci 2005; 20:659-662.

104. Carette S, Marcoux S, Truchon R, Grondin C, Gagnon J, Allard Y, Latulippe M. A controlled trial of corticosteroid injections into facet joints for chronic low back pain. N Engl J Med 1991; 325:1002-1007.

105. Lilius G, Laasonen EM, Myllynen P, Harilainen A, Gronlund G. Lumbar facet joint syndrome: a randomized clinical trial. J Bone Joint Surg (Br) 1989; 71:681-684.

106. Marks RC, Houston T, Thulbourne T. Facet joint injection and facet nerve block: a randomized comparison in 86 patients with chronic low back pain. Pain 1992; 49:325-328.

107. Nash TP. Facet joints. Intraarticular steroids or nerve blocks? Pain Clinic 1990; 3:77-82.

108. Barnsley L, Lord SM, Wallis BJ, Bogduk $N$. Lack of effect of intraarticular corticosteroids for chronic pain in the cervical zygapophyseal joints. N Engl J Med 1994; 330:1047-1050.

109. Murtagh FR. Computed tomography and fluoroscopy guided anesthesia and steroid injection in facet syndrome. Spine 1988; 13:686-689.

110. Destouet JM, Gilula LA, Murphy WA, Monsees B. Lumbar facet joint injection: lindication, technique, clinical correlation, and preliminary results. Radiology 1982; 145:321-325.

111. Lynch MC, Taylor JF. Facet joint injection for low back pain. A clinical study. J Bone Joint Surg Br 1986; 68:138-141.

112. Lippitt $A B$. The facet joint and its role in spine pain. Management with facet joint injections. Spine 1984; 9:746-750.

113. Lau LS, Littlejohn GO, Miller MH. Clinical evaluation of intraarticular injec- 
tions for lumbar facet joint pain. Med J Aust 1985; 143:563-565.

114. Schulte TL, Pietila TA, Heidenreich J, Brock M, Stendel R. Injection therapy of lumbar facet syndrome: a prospective study. Acta Neurochir (Wien) 2006; 148:1165-1172.

115. Folman Y, Livshitz A, Shabat S, Gepstein R. Relief of chronic cervical pain after selective blockade of zygapophyseal joint. Harefuah 2004; 143:339341, 391.

116. Manchikanti L, Damron K, Cash K Manchukonda R, Pampati V. Therapeutic cervical medial branch blocks in managing chronic neck pain: a preliminary report of a randomized, double-blind, controlled trial. Clinical trial Ncto033272. Pain Physician 2006; 9: 333-346.

117. Manchikanti L, Damron KS, Cash KA, Manchukonda R, Pampati V. Evaluation of lumbar facet joint nerve blocks in the management of chronic low back pain: a preliminary report of a randomized, double-blind controlled trial. Clin ical Trial NCTo0355914. 2007; in press.

118. Manchikanti L, Manchikanti KN, Manchukonda R, Pampati V, Cash K. Evaluation of therapeutic thoracic medial branch block effectiveness in chronic thoracic pain: A prospective outcome study with minimum 1-year follow up. Pain Physician 2006; 9:97-105.

119. Manchikanti L, Pampati VS, Bakhit C, Rivera JJ, Beyer CD, Damron KS, Barnhill RC. Effectiveness of lumbar facet joint nerve blocks in chronic low back pain: a randomized clinical trial. Pain Physician 2001; 4:101-117.

120. Manchikanti KN, Pampati V, Damron KS, McManus CD. A double-blind, controlled evaluation of the value of Sarapin in neural blockade. Pain Physician 2004; 7:59-62.

121. Manchikanti L, Manchikanti KN, Damron KS, Pampati V. Effectiveness of cervical medial branch blocks in chronic neck pain: a prospective outcome study. Pain Physician 2004; 7:195-201.

122. Manchikanti L, Singh V, Pampati V. Are diagnostic lumbar medial branch blocks valid? Results of 2-year follow up. Pain Physician 2003; 6:147-153.

123. van Wijk RMAW, Geurts JWM, Wynne HJ, Hammink E, Buskens E, Lousberg R, Knape JTA, Groen GJ. Radiofrequency denervation of lumbar facet joints in the treatment of chronic back pain. A Randomized, double-blind, sham lesion-controlled trial. Clin J Pain 2005; 21:335-344.
124. Bogduk J. Lumbar radiofrequency neurotomy (Commentary.) Clin J Pain 2005; 21:335-344.

125. Haspeslagh SR, Van Suijlekom HA, Lame IE, Kessels AG, van Kleef M, Weber WE. Randomised controlled trial of cervical radiofrequency lesions as a treatment for cervicogenic headache [ISRCTNo7444684]. BMC Anesthesiol 2006; 6:1.

126. Barnsley L. Percutaneous radiofrequency neurotomy for chronic neck pain: outcomes in a series of consecutive patients. Pain Med 2005; 6:282-286.

127. Shin WR, Kim HI, Shin DG, Shin DA. Radiofrequency neurotomy of cervical medial branches for chronic cervicobrachialgia. J Korean Med Sci 2006; 21:119-125.

128. Birkenmaier C, Veihelmann A, Trouillier H, Hausdorf J, Devens C, Wegener $B$, Jansson V, von Schulze Pellengahr C. Percutaneous cryodenervation of lumbar facet joints: a prospective clinical trial. Int Orthop 2006; Aug 23. [Epub ahead of print]

129. Staender M, Maerz U, Tonn JC, Steude U. Computerized tomography-guided kryorhizotomy in 76 patients with lumbar facet joint syndrome. I Neurosurg Spine 2005; 3:444-449.

130. Mogalles AA, Dreval ON, Akatov OV, Kuznetsov AV, Rynkov IP, Plotnikov VM, Minaev VP. Percutaneous laser denervation of the zygapophyseal joints in the pain facet syndrome. Zh Vopr Neirokhir Im N N Burdenko 2004; 1:20-25.

131. Martinez-Suarez JE, Camblor L, Salva $\mathrm{S}$, De Jongh WA. Thermocoagulation of lumbar facet joints. Experience in 252 patients. Revista de la Sociedad Espanola del Dolor 2005; 12:425-428.

132. Lindner R, Sluijter ME, Schleinzer W. Pulsed radiofrequency treatment of the lumbar medial branch for facet pain: a retrospective analysis. Pain Med 2006; 7:435-439.

133. Mikeladze G, Espinal R, Finnegan R, Routon J, Martin D. Pulsed radiofrequency application in treatment of chronic zygapophyseal joint pain. Spine J 2003; 3:360-362.

134. Lord SM, Barnsley L, Wallis BJ, McDonald GJ, Bogduk N. Percutaneous radiofrequency neurotomy for chronic cervical zygapophyseal joint pain. $N$ Eng J Med 1996; 335:1721-1726.

135. van Kleef M, Barendse GAM, Kessels A, Voets HM, Weber WE, de Lange S. Randomized trial of radiofrequency lumbar facet denervation for chronic low back pain. Spine 1999; 24:1937-1942.
136. Sapir D, Gorup JM. Radiofrequency medial branch neurotomy in litigant and non-litigant patients with cervical whiplash. Spine 2001; 26:E268-E273.

137. McDonald G), Lord SM, Bogduk N. Long-term follow-up of patients treated with cervical radiofrequency neurotomy for chronic neck pain. Neurosurgery 1999; 45:61-68.

138. Tzaan WC, Tasker RR. Percutaneous radiofrequency facet rhizotomy - experience with 118 procedures and reappraisal of its value. Can J Neurol Sci 2000; 27:125-130.

139. Schaerer JP. Radiofrequency facet rhizotomy in the treatment of chronic neck and low back pain. Int Surg 1978; 63:53-59.

140. Dreyfuss P, Halbrook B, Pauza K, Joshi A, McLarty J, Bogduk N. Efficacy and validity of radiofrequency neurotomy for chronic lumbar zygapophysial joint pain. Spine 2000; 25:1270-1277.

141. Schofferman J, Kine G. Effectiveness of repeated radiofrequency neurotomy for lumbar facet pain. Spine 2004; 29:2471-2473

142. Vad VB, Cano WG, Basrai D, Lutz GE, Bhat AL. Role of radiofrequency denervation in lumbar zygapophyseal joint synovitis in baseball pitchers: a clinical experience. Pain Physician 2003; 6:307-312.

143. Gallagher J, Vadi PLP, Wesley JR. Radiofrequency facet joint denervation in the treatment of low back pain: a prospective controlled double-blind study to assess efficacy. Pain Clinic 1994; 7:193-198.

144. LeClaire $R$, Fortin L, Lambert $R$, Bergeron YM, Rossignol M. Radiofrequency facet joint denervation in the treatment of low back pain: a placebocontrolled clinical trial to assess efficacy. Spine 2001; 26:1411-1417.

145. Sanders M, Zuurmond WWA. Percutaneous intraarticular lumbar facet joint denervation in the treatment of low back pain: a comparison with percutaneous extra-articular lumbar facet denervation. Pain Clinic 1999; 11:329-335.

146. Buijs EJ, van Wijk RM, Geurts JW, Weeseman RR, Stolker RJ, Groen GG. Radiofrequency lumbar facet denervation: a comparative study of the reproducibility of lesion size after 2 current radiofrequency techniques. Reg Anesth Pain Med 2004; 9:400-407.

147. van Kleef $M$, Liem L, Lousberg R, Barendse G, Kessels F, Sluijter M. Radiofrequency lesion adjacent to the dorsal root ganglion for cervicobrachial 
pain: a prospective double blind randomized study. Neurosurgery 1996; 38:1127-1131.

148. Slappendel R, Crul BJ, Braak GJ, Geurts JW, Booij LH, Voerman VF, de Boo T. The efficacy of radiofrequency lesioning of the cervical spinal dorsal root ganglion in a double blinded randomized study: no difference between $40^{\circ} \mathrm{C}$ and $67^{\circ} \mathrm{C}$ treatments. Pain 1997; 73:159-163.

149. North RB, Han M, Zahurak M, Kidd DH. Radiofrequency lumbar facet denervation: analysis of prognostic factors. Pain 1994; 57:77-83.

150. Stolker RJ, Vervest AC, Groen GJ. Percutaneous facet denervation in chronic thoracic spinal pain. Acta Neurochir 1993; 122:82-90.

151. Bogduk N. Percutaneous radiofrequency cervical medial branch neurotomy. In: Practice Guidelines for Spinal Diag. nostic and Treatment Procedures, 1st edition. International Spine Intervention Society, 2004, pp 249-284.

152. Weingarten TN, Hooten WM, Huntoon MA. Septic facet joint arthritis after a corticosteroid facet injection. Pain Med 2006; 7:52-56.

153. Heckman JG, Maihofner C, Lanz S, Rauch C, Neundorfer B. Transient tetraplegia after cervical facet joint injection for chronic neck pain administered without imaging guidance. Clin Neurol Neurosurg 2006; 108:709-711.

154. Smith M, Ferretti G, Mortazavi S. Radiographic changes induced after cervical facet radiofrequency denervation. Spine J 2005; 5: 668-671.

155. Huntoon MA. Anatomy of the cervical intervertebral foramina: vulnerable arteries and ischemic neurologic inju- ries after transforaminal epidural injections. Pain 2005; 117:104-111.

156. Muffolerro AJ, Nader R, Westmark RM, Nauta HJ, Garges KJ, Hadjipavlou AG. Hematogenous pyogenic facet joint in fection of the subaxial cervical spine: a report of two cases and review of the literature. J Neurosurg Spine 2001; 95:135-138.

157. Doita M, Nishida K, Miyamoto H, Yoshiya S, Kurosaka M, Nabeshima Y. Septic arthritis of bilateral lumbar facet joints: report of a case with MRI findings in the early stage. Spine 2003; 28:E198-202.

158. Orpen NM, Birch NC. Delayed presentation of septic arthritis of a lumbar facet joint after diagnostic facet joint injection. I Spinal Disord Tech 2003; 16:285-287.

159. Alcock E, Regaard A, Browne J. Facet joint injection: a rare form cause of epidural abscess formation. Pain 2003; 103:209-210.

160. Magee M, Kannangara S, Dennien B, Lonergan R, Emmett L, van der Wall $H$. Paraspinal abscess complicating facet joint injection. Clin Nucl Med 2000; 25:71-73.

161. Windsor RE, Pinzon EG, Gore HC. Complications of common selective spinal injections: prevention and management. Am J Orthop 2000; 29:759-770.

162. Marks R, Semple A): Spinal anaesthesia after facet joint injection. Anaesthesia 1988; 43:65-66.

163. Cook NJ, Hanrahan P, Song S. Paraspinal abscess following facet joint injection. Clin Rheumatol 1999; 18:52-53.

164. Manchikanti L, Cash K, Moss T, Rivera JJ, Pampati V. Risk of whole body radia- tion exposure and protective measures in fluoroscopically guided interventional techniques: a prospective evaluation. BMC Anesthesiol 2003; 3:2.

165. Berrigan T. Chemical meningism after lumbar facet joint block. Anesthesia 1992; 7:905-906.

166. Thomson SJ, Lomax DM, Collett BJ. Chemical meningism after lumbar facet joint nerve block with local anesthetic and steroids. Anesthesia 1993; 46:563-564.

167. Raj PP, Shah RV, Kay AD, Denaro S, Hoover JM. Bleeding risk in interventional pain practice: assessment, management, and review of the literature. Pain Physician 2004; 7:3-52.

168. Dreyfuss P, Kaplan M, Dreyer SJ. Zygapophyseal joint injection techniques in the spinal axis. In: Lennard T (ed.) Pain Procedures in Clinical Practice, 2nd ed. Hanley \& Belfus, Inc., Philadelphia, 2000, pp 276-308.

169. Manchikanti L. Role of neuraxial steroids in interventional pain management. Pain Physician 2002; 5:182-199.

170. Manchikanti L, Pampati V, Beyer C, Damron KS, Cash KA, Moss TL. The effect of neuraxial steroids on weight and bone mass density: a prospective evaluation. Pain Physician 2000; 3:357-366.

171. Kornick CA, Kramarich SS, Lamer TJ, Sitzman BT. Complications of lumbar facet radiofrequency denervation. Spine 2004; 29:1352-1354.

172. Windsor RE, Storm S, Sugar R. Prevention and management of complications resulting from common spinal injections. Pain Physician 2003; 6:473-484. 
